\title{
SOME PROPERTIES OF TRIGONOMETRIC SERIES WHOSE TERMS HAVE RANDOM SIGNS
}

\author{
Dedicated to Professor Hugo Steinhaus for his 65th Birthday
}

BY

\section{R. SALEM and A. ZYGMUND}

Trigonometric series of the type

$$
\sum_{1}^{\infty} \varphi_{n}(t)\left(a_{n} \cos n x+b_{n} \sin n x\right),
$$

where $\left\{\varphi_{n}(t)\right\}$ denotes the system of Rademacher functions, have been extensively studied in order to discover properties which belong to "almost all" series, that is to say which are true for almost all values of $t .^{1}$ We propose here to add some new contributions to the theory.

\section{CHA P T E R I}

\section{Weighted Means of Ortho-normal Functions}

1. Let $\varphi_{1}, \varphi_{2}, \ldots, \varphi_{n}, \ldots$ be a system of functions of $x$, ortho-normal in an interval $(a, b)$, and let $\gamma_{1}, \gamma_{2}, \ldots, \gamma_{n}, \ldots$ be a sequence of non-negative constants such that

$$
S_{n}=\gamma_{1}+\gamma_{2}+\cdots+\gamma_{n}
$$

increases indefinitely as $n$ tends to $+\infty$. Under what conditions does the mean

$$
R_{n}(x)=\frac{\gamma_{1} \varphi_{1}(x)+\gamma_{2} \varphi_{2}(x)+\cdots+\gamma_{n} \varphi_{n}(x)}{\gamma_{1}+\gamma_{2}+\cdots+\gamma_{n}}
$$

tend to zero almost everywhere ${ }^{2}$ as $n \rightarrow \infty$ ?

1 Cf., in particular, Paley and Zygmund, Proc. Cambridge Phil. Soc., 26 (1930), pp. 337-357 and 458-474, and 28 (1932), pp. 190-205.

2 We write briefly $R_{n}(x) \rightarrow 0$ p.p. ("presque partout"). 
It has been proved that, if $\varphi_{n}=e^{i n x}$, then $R_{n}(x) \rightarrow 0$ p.p., provided $\gamma_{n}=O(1)$. The proof is applicable without change to any ortho-normal uniformly bounded system. As it was observed in the paper, some condition on the $\gamma_{n}$ is indispensable, since, e.g.

$$
2^{-n} \sum_{1}^{n} 2^{k} e^{i k x}
$$

does not tend to zero almost everywhere as $n \rightarrow \infty$.

More recently, Hill and Kakutani have raised the question whether $R_{n}(x) \rightarrow 0$ p.p. if $\left\{\varphi_{n}\right\}$ is the Rademacher system, the sequence $\gamma_{n}$ is monotonically increasing and $\gamma_{n}=o\left(S_{n}\right)$. The answer has been proved by several authors to be negative.2

Here we propose to give a sufficient condition in order that $R_{n}(x) \rightarrow 0$ p.p., when $\left\{\varphi_{n}\right\}$ is any uniformly bounded ortho-normal system in $(a, b)$ and to prove, by the consideration of the trigonometric system, that this condition is the best possible one.

Let us observe first of all that the condition $\gamma_{n}=o\left(S_{n}\right)$ is trivially necessary in order that $R_{n}(x) \rightarrow 0$ p.p. For $\gamma_{n} / S_{n}=\int_{a}^{b} R_{n} \varphi_{n} d x$, and the uniform boundedness of the $\varphi_{n}$ implies that $R_{n} \varphi_{n} \rightarrow 0$ p.p., boundedly, whence $\gamma_{n} / S_{n} \rightarrow 0$.

As we shall see, the condition $\gamma_{n}=o\left(S_{n}\right)$ is, in general, not sufficient. Let us note, however, that in the case $\gamma_{n}=e^{i n x}$, if the sequence $\left\{\gamma_{n}\right\}$ is monotonic and $\gamma_{n}=o\left(S_{n}\right)$, then $R_{n}(x) \rightarrow 0$ everywhere, except for $x=0$. This follows from an application of summation by parts to the numerator of $R_{n}$.

2. (1.2.1) Theorem. Let $\left\{\varphi_{n}\right\}$ be an ortho-normal and uniformly bounded system in $(a, b)$, and let $\left|\varphi_{n}\right| \leq M$. Let $\omega(u)$ be a monotonically increasing function of $u$ such that $u / \omega(u)$ increases to $+\infty$ and such that $\sum 1 / k \omega(k)<\infty$. Then $R_{n}(x) \rightarrow 0$ p.p., provided $\gamma_{n}=O\left\{S_{n} / \omega\left(S_{n}\right)\right\}$.

Proof. Let us recall first that, if we set

$$
\gamma_{n}^{*}=\operatorname{Max}_{m} \gamma_{m} \quad(1 \leq m \leq n)
$$

then also $\gamma_{n}^{*}=O\left\{S_{n} / \omega\left(S_{n}\right)\right\}$. For we have $\gamma_{n}^{*}=\gamma_{p}$, where $p=p(n) \leq n$ is non-decreasing. Let $Q_{n}=S_{n} / \omega\left(S_{n}\right)$. Then p. 333 .

1 Cf. R. SAlem, The absolute convergence of trigonometric series, Duke Math. Journal, 8 (1941),

2 See Tamotsu Tsuchikura, Proc. of the Japan Academy, 27 (1951), pp. 141-145, and the re. sults quoted there, especially Maruyama's result. 


$$
\frac{\gamma_{p}}{Q_{n}}=\frac{\gamma_{p}}{Q_{p}} \cdot \frac{Q_{p}}{Q_{n}} \leq \frac{\gamma_{p}}{Q_{p}}
$$

and our assertion follows.

Consequently one also has

$$
\frac{\sum_{1}^{N} \gamma_{n}^{2}}{S_{N}^{2}}=O\left\{\frac{1}{\omega\left(S_{N}\right)}\right\}
$$

Let us fix a number $\theta>1$ and let $N_{j}$ be the first integer such that

$$
\theta^{j} \leq S_{N_{j}}<\theta^{j+1}
$$

$N_{f}$ always exists for $j$ large enough. For otherwise there would exist arbitrarily large integers $j$ such that for a suitable $m$ we would have $\theta^{j} \leq S_{m}<\theta^{j+1}$ and simultaneously $S_{m: 1}<\theta^{j 1}$. This would imply

$$
\gamma_{m}>\theta^{j}-\theta^{j \cdots 1}, \quad \frac{\gamma_{m}}{S_{m}}>\frac{\theta^{j}-\theta^{j-1}}{\theta^{j+1}}=\frac{\theta-1}{\theta^{2}},
$$

contradicting the assumption $\gamma_{n}=o\left(S_{n}\right)$.

Now, by (1.2.2),

$$
\sum_{j} \int_{a}^{b}\left|R_{N_{j}}\right|^{2} d x=\sum_{j} O\left\{\frac{1}{\omega\left(\theta^{j}\right)}\right\}<\infty
$$

by the hypothesis $\sum 1 / k \omega(k)<\infty$. Hence $R_{N_{j}} \rightarrow 0$ p.p.

Let now $N_{j} \leq m<N_{j+1}$. One has

$$
R_{m}=\frac{\sum_{1}^{N_{j}} \gamma_{n} \varphi_{n}}{S_{m}}+\frac{\sum_{N_{j}+1}^{m} \gamma_{n} \varphi_{n}}{S_{m}} .
$$

The first ratio tends to zero almost everywhere since $S_{m} \geq S_{N_{j}}$; the second one has absolute value less than

$$
M \frac{\sum_{N_{j+1}}^{N_{j+1}} \gamma_{n}}{S_{N_{j}}}=M \frac{S_{N_{j+1}}-S_{N_{j}}}{S_{N_{j}}}<M \frac{\theta^{j+2}-\theta^{j}}{\theta^{j}}=M\left(\theta^{2}-1\right) .
$$

It follows that

$$
\lim \sup \left|R_{m}\right| \leq M\left(\theta^{2}-1\right)
$$

almost everywhere. Since $\theta$ can be taken arbitrarily close to 1 , the theorem follows. 
3. We shall now show that the preceding theorem no longer holds if we allow $\Sigma 1 / k \omega(k)$ to diverge. This will follow from the following

(1.3.1) Theorem. Given any function $\omega(t)$ increasing to $+\infty$ with $t$ and such that $\int_{t \omega(t)}^{\infty} \frac{d t}{t \omega}=\infty$, and assuming for the sake of simplicity that $\omega(t) / \log t$ is monotonic, there exists a sequence $\gamma_{n}$ such that $\gamma_{n}=O\left\{S_{n} / \omega\left(S_{n}\right)\right\}$ and that

$$
\lim \sup \left|\frac{\gamma_{1} e^{i x}+\cdots+\gamma_{n} e^{i n x}}{\gamma_{1}+\cdots+\gamma_{n}}\right|=1
$$

almost everywhere.

We shall first prove two lemmas.

(1.3.2) Lemma. Let $\left\{m_{q}\right\}$ be an increasing sequence of integers such that $m_{a} / q$ is monotonic and $\Sigma 1 / m_{\mathrm{q}}=\infty$. Then

$$
\lim \sup \frac{1}{m_{a}}\left|\frac{\sin q m_{a} x}{\sin q x}\right|=1
$$

almost everywhere.

Proof. By a well-known theorem of Khintchine, the conditions imposed on the sequence $\left\{m_{\mathbf{q}}\right\}$ imply that for almost every $x$ there exist infinitely many integers $p$ and $q$ such that

$$
\left|\frac{x}{2 \pi}-\frac{p}{q}\right|=o\left(\frac{1}{q m_{q}}\right)
$$

Hence, fixing an $x$ having this property, one has

$$
\begin{aligned}
& |q x-2 \pi p|=\varepsilon_{q} / m_{q} \\
& \left|q m_{q} x-2 \pi p m_{q}\right|=\varepsilon_{q}
\end{aligned}
$$

for infinitely many $p, q$, with $\varepsilon_{q} \rightarrow 0$. Hence also, for infinitely many values of $q$,

$$
\frac{1}{m_{q}} \frac{\sin q m_{q} x}{\sin q x}=\frac{1}{m_{q}} \frac{\sin \varepsilon_{q}}{\sin \left(\varepsilon_{q} / m_{q}\right)},
$$

and the lemma follows.

As a simple special case we get that $\lim \sup \left|\frac{\sin q^{2} x}{q \sin q x}\right|=1$ p.p.

Remark. If $\Sigma 1 / m_{q}<\infty$, one has $\lim \frac{\sin q m_{q} x}{m_{a} \sin q x}=0$ p.p. This follows immediately from the fact that 


$$
\frac{1}{m_{a}^{2}} \int_{0}^{2 \pi}\left(\frac{\sin q m_{a} x}{\sin q x}\right)^{2} d x=O\left(\frac{1}{m_{a}}\right)
$$

(1.3.3) Lemma. Let $\left\{m_{q}\right\}$ be an increasing sequence of integers such that $m_{\sigma} / q$ is monotonic and that $\Sigma 1 / m_{q}=\infty$. Let us set, for each positive integer $q$,

and

$$
h=h(q)=q m_{q}+1, \quad k=k(q)=(q+1) m_{q}
$$

$$
F_{Q}(x)=e^{h q i x}+e^{(h+1) q i x}+\cdots+e^{k q i x} .
$$

Then

$$
\lim \sup \left|F_{Q}(x)\right| / m_{q}=1
$$

almost everywhere.

Proof. One has

$$
\begin{gathered}
F_{q}=e^{h q i x} \frac{e^{(k-h+1) q i x}-1}{e^{q i x}-1}, \\
\left|F_{a}\right|=\left|\frac{\sin \frac{1}{2}(k-h+1) q x}{\sin \frac{1}{2} q x}\right|=\left|\frac{\sin \frac{1}{2} q m_{q} x}{\sin \frac{1}{2} q x}\right|,
\end{gathered}
$$

and it is enough to apply the preceding lemma.

Prool of Theorem (1.3.1). It will be sufficient to consider the stronger case in which $\omega(t) / \log t$ increases to $\infty$. We write $\omega(t)=\log t \lambda(\log t)$ where $\lambda(u)$ increases to $\infty$ with $u$. Observe that

$$
\int^{\infty} \frac{d u}{u \lambda(u)}=\int^{\infty} \frac{d t}{t \log t \lambda(\log t)}=\int^{\infty} \frac{d t}{t \omega(t)}=\infty .
$$

Hence we can find an increasing sequence of integers $m_{q}$ such that $m_{a} / q \lambda(q)$ increases to $\infty$ and that $\Sigma 1 / m_{q}=\infty$.

By $\varphi(q)$ we shall denote a function of $q$ increasing to $\infty$ as slowly as we wish and which we shall determine later on.

Let us now set, for each integer $q$, as in Lemma (1.3.3),

and let

$$
h=h(q)=q m_{q}+1, k=k(q)=(q+1) m_{a}
$$

$$
\begin{aligned}
& \gamma_{n}=\frac{e^{\sigma \varphi(q)}}{m_{a}}=c_{q} \text { for } n=\nu q \quad(h \leq \nu \leq k) \\
& \gamma_{n}=0 \text { for } n \neq \nu q \quad(h q<n<k q) .
\end{aligned}
$$


We note that, $F_{q}$ being defined as in Lemma (1.3.3), there is no overlapping of terms of $F_{a}$ and $F_{q+1}$, since $q k(q)<(q+1) h(q+1)$.

Let us now write

$$
\varrho_{q}=\frac{c_{1} F_{1}+c_{2} F_{2}+\cdots+c_{q} F_{q}}{c_{1} m_{1}+c_{2} m_{2}+\cdots+c_{a} m_{q}}
$$

This ratio is equal to $R_{n}(x)$ for $n=q k(q)$.

One has

$$
\underline{\varrho}_{q}=\frac{\left(c_{1} F_{1}+\cdots+c_{q-1} F_{q-1}\right) e^{-q \varphi(q)}+F_{q} / m_{Q}}{\left(c_{1} m_{1}+\cdots+c_{q-1} m_{q-1}\right) e^{-q \varphi(Q)}+1}
$$

and since

$$
\left|c_{1} F_{1}+\cdots+c_{q}{ }_{1} F_{q, 1}\right| \leq c_{1} m_{1}+\cdots+c_{q-1} m_{q, 1}=\sum_{1}^{q-1} e^{k \varphi(k)}=o\left(e^{Q \varphi(q)}\right),
$$

one has

$$
\lim \sup \left|\varrho_{a}\right|=\lim \sup \left|F_{a}\right| / m_{q}=1 .
$$

On the other hand, if $e^{n i x}$ occurs in $F_{q}$,

Hence

$$
\gamma_{n}=\frac{e^{q \varphi(\theta)}}{m_{q}} \text { and } S_{n}>\sum_{1}^{Q} e^{1} e^{k \varphi(k)}>e^{(\theta 1) \varphi(\theta 1)}
$$

$$
\gamma_{n} / S_{n}<m_{q}{ }^{1}\left[e^{q \varphi(q)}\left(\begin{array}{lll}
q & 1) \varphi(q \cdot 1)
\end{array}\right] .\right.
$$

Since $m_{\mathfrak{q}} / q \lambda(q)$ increases to $\infty$, we can choose $\varphi(q)$ increasing slowly enough in order to have

Therefore,

$$
\frac{m_{a}}{q \varphi(q) \lambda[q \varphi(q)]}>e^{q \varphi(q) \cdot(q \cdot 1) \varphi(e-1)}
$$

$$
\frac{\gamma_{n}}{S_{n}} \leq \frac{1}{q \varphi(q) \lambda[q \varphi(q)]} .
$$

But

$$
S_{n}<\sum_{1}^{Q} e^{k \varphi(k)} \sim e^{Q \varphi(q)}
$$

It follows that

$$
\frac{\gamma_{n}}{S_{n}}=O\left[\frac{1}{\log S_{n} \lambda\left(\log S_{n}\right)}\right]=O\left[\frac{1}{\omega\left(S_{n}\right)}\right]
$$

which proves the theorem. 
4. As we have mentioned above, the case of Rademacher functions has been completely investigated by Maruyama and Tsuchikura. We give here different proofs of their two results.

(1.4.1) Theorem. Let $\varphi_{1}, \varphi_{2}, \ldots, \varphi_{n}, \ldots$ be the system of Rademacher functions. Then $\gamma_{n}=o\left\{S_{n} / \log \log S_{n}\right\}$ is a sufficient condition in order that $R_{n} \rightarrow 0$ p.p.

We have, as in Section 2,

$$
\frac{\sum_{1}^{N} \gamma_{n}^{2}}{S_{N}^{2}}=o\left(\frac{1}{\log \log S_{N}}\right)=\frac{\varepsilon_{N}}{\log \log S_{N}},
$$

where $\varepsilon_{N} \rightarrow 0$. Since for the Rademacher functions one has

$$
\int_{0}^{1}\left(\sum_{1}^{N} \gamma_{n} \varphi_{n}\right)^{2 k} d x \leq k^{k}\left(\sum_{1}^{N} \gamma_{n}^{2}\right)^{k} \quad(k=1,2, \ldots)
$$

it follows that, $A$ being a positive constant,

$$
\int_{0}^{1}\left(A R_{N}\right)^{2 k} d x \leq A^{2 k} \frac{k^{k}\left(\sum_{1}^{N} \gamma_{n}^{2}\right)^{k}}{S_{N}^{2 k}}=\left(\frac{A^{2} k \varepsilon_{N}}{\log \log S_{N}}\right)^{k} .
$$

Let us define $k=k(N)$ to be the integral part of $\left(e A^{2} \varepsilon_{N}\right)^{1} \log \log S_{N}$. We get

$$
\int_{0}^{1}\left(A R_{N}\right)^{2 \kappa} d x \leq\left(\frac{1}{e}\right)^{\frac{\log \log S_{N}}{e A^{2} \varepsilon_{N}}-1} .
$$

Let us now take $\theta>1$ and define, as in Section 2, a sequence $\left\{N_{j}\right\}$ such that $\theta^{f} \leq S_{N_{j}}<\theta^{j+1}$. Let $k_{j}=k\left(N_{j}\right)$. One has, for $j$ large enough,

$$
\frac{1}{e A^{2} \varepsilon_{N_{j}}}>2 \text {. }
$$

Hence

$$
\int_{0}^{1}\left(A R_{N_{j}}\right)^{2 k_{j}} d x=O\left(j^{-2}\right)
$$

so that

$$
\sum_{j}\left(A R_{N_{j}}\right)^{2 k_{j}}<\infty \text { p.p. }
$$


It follows that $\lim \sup R_{N_{j}} \leq A^{-1}$ p.p., and so also that $\lim R_{N_{j}}=0$ p.p., since $A$ can be taken arbitrarily large. From here we proceed, without change, as in Section 2.

Remark 1. An alternate proof could be given using an extension of the Law of the Iterated Logarithm pertaining to the case of Rademacher functions, but the proof given above is simpler and more direct.

Remark 2. The theorem can obviously be extended to other orthogonal systems for which the inequality of the type (1.4.2) holds, e.g. to certain types of independent functions, and also lacunary trigonometric functions, such as $\cos 2^{n} x$.

5. (1.5.1) Theorem. Theorem (1.4.1) becomes false if we replace in the assumption the order " $O$ " by " $O$ ". More precisely, there exists a sequence $\left\{\gamma_{n}\right\}$ such that

$$
\gamma_{n}=O\left(S_{n} / \log \log S_{n}\right)
$$

and such that $\lim \sup R_{n}>\propto$ p.p., $\alpha$ being any fixed constant less than 1 .

(1.5.2) Lemma. Let $\varphi_{1}, \varphi_{2}, \ldots$ be the system of Rademacher functions and let $\left\{m_{p}\right\}$ be an increasing sequence of integers such that

$$
\Delta_{p}=m_{\mathcal{D}}-m_{p} \quad \leq \frac{\log p}{\log 2} .
$$

Then, writing

$$
F_{p}=\sum_{m_{p-1}+1}^{m_{p}} \varphi_{k}
$$

one has

$$
\lim \sup \boldsymbol{F}_{p} / \Delta_{\mathfrak{p}}=1 \text { p.p. }
$$

The set in which $F_{p}=\Delta_{p}$ has measure $2 \Delta_{p}$. Hence the set where $F_{p} \neq \Delta_{p}$ is of measure $1-2^{-\Delta_{p}}$; and the set $E_{p}$ where $F_{p}, F_{p+1}, \ldots$ are all different from $\Delta_{p}$, $\Delta_{p, 1}, \ldots$, respectively, is of measure

$$
\prod_{q-p}^{\infty}\left(1-2^{-\Delta_{q}}\right)=0
$$

by the hypothesis on $\Delta_{q}$. If, for a given $x, \lim \sup F_{p} / \Delta_{p}<1$, then, clearly, $x$ belongs to some $E_{p}$. Since the sets $E_{p}$ are all of measure zero, the lemma follows.

We are now able to prove Theorem (1.5.1). Preserving the notation of the lemma, we take a sequence of integers $m_{p}$ satisfying the conditions

$$
\frac{1}{3} \log p<m_{p}-m_{p-1}<\frac{1}{2} \log p
$$


so that the condition $\Delta_{p} \leq \log p / \log 2$ is satisfied. Let us now take, with $A>1$,

$$
\gamma_{n}=A^{p} / \Delta_{p}=c_{p}, \text { for } m_{p-1}<n \leq m_{p}
$$

and consider the ratio

$$
\varrho_{p}=\frac{c_{1} F_{1}+c_{2} F_{2}+\cdots+c_{p} F_{p}}{c_{1} \Delta_{1}+c_{2} \Delta_{2}+\cdots+c_{p} \Delta_{p}}
$$

which is equal to $R_{n}(x)$ for $n=m_{p}$. One has

$$
\varrho_{p}=\frac{\left(c_{1} F_{1}+\cdots+c_{p-1} F_{p-1}\right) A^{-p}+F_{p} \Delta_{p}^{-1}}{\left(c_{1} \Delta_{1}+\cdots+c_{p-1} \Delta_{p-1}\right) A^{-p}+1}
$$

Now,

$$
\left|A^{-p}\left(c_{1} F_{1}+\cdots+c_{p-1} F_{p-1}\right)\right| \leq A^{-p}\left(c_{1} \Delta_{1}+\cdots+c_{p-1} \Delta_{p-1}\right)=A^{-p} \sum_{1}^{p-1} A^{k}
$$

Hence, if $A$ is large enough, using the lemma,

$$
\lim \sup \varrho_{p}>\alpha \text { p.p. }
$$

On the other hand, if $\varphi_{n}$ occurs in $F_{p}$, then

$$
\begin{gathered}
\gamma_{n}=A^{p} \Delta_{p}^{-1}<3 . A^{p} / \log p, \\
S_{m_{p-1}}<S_{n} \leq S_{m_{p}},
\end{gathered}
$$

so that $\log \log S_{n} \sim 2 \log p$, and $\gamma_{n}=O\left\{S_{n} / \log \log S_{n}\right\}$.

6. To theorems about the partial sums of divergent series often correspond theorems about the remainders of convergent series, and the results of this chapter admit of such extensions. We shall be satisfied with stating here the following analogue of Theorem (1.2.1), in which the functions $\omega(u)$ and $\varphi_{n}$ have the same meaning as there.

(1.6.1) Theorem. If $\gamma_{1}+\gamma_{2}+\cdots+\gamma_{n}+\cdots$ is a convergent series with positive terms, and if $S_{n}$ denotes the remainder $\gamma_{n}+\gamma_{n+1}+\cdots$, then

$$
\bar{R}_{n}(x)=\frac{\gamma_{n} \varphi_{n}+\gamma_{n+1} \varphi_{n+1}+\cdots}{\gamma_{n}+\gamma_{n+1}+\cdots}
$$

tends to 0 p.p., provided $\gamma_{n}=O\left\{S_{n} / \omega\left(1 / S_{n}\right)\right\}$.

The proof is identical with that of Theorem (1.2.1). 


\section{CHAPTER I I}

\section{The Law of the Iterated Logarithm}

1. As proved by Kolmogoroff, the law can be stated as follows. Let

$$
z_{1}, z_{2}, \ldots, z_{n}, \ldots
$$

be independent random variables with vanishing mean values and with dispersions $b_{1}, b_{2}, \ldots, b_{n}, \ldots$, respectively. Let

$$
S_{N}=\sum_{1}^{N} z_{k}, B_{N}^{2}=\sum_{1}^{N} b_{k}
$$

Under the assumptions

$$
B_{N}^{2} \rightarrow \infty,\left|z_{N}\right| \leq m_{N}=o\left\{\frac{B_{N}^{2}}{\log \log B_{N}}\right\}^{\frac{1}{2}}
$$

one has, with probability 1 ,

$$
\lim \sup _{\left(2 B_{N}^{2} \log \log B_{N}\right)^{\frac{1}{t}}=1 .}^{S_{N}}
$$

The result $\lim$ sup $\leq 1$ has been extended by the authors to the case in which the series of random variables is replaced by a lacunary trigonometric series

$$
\sum\left(a_{k} \cos n_{k} x+b_{k} \sin n_{k} x\right),
$$

with $n_{k+1} / n_{k}>q>1 .^{1}$ Here we propose to give a theorem equivalent to Kolmogoroff's, valid for almost all trigonometric series of the type $(0.1)$.

(2.1.2) Theorem. Let us consider the series

$$
\sum_{1}^{\infty} \varphi_{n}(t)\left(a_{n} \cos n x+b_{n} \sin n x\right)=\sum_{1}^{\infty} \varphi_{n}(t) A_{n}(x)
$$

where $\left\{\varphi_{n}(t)\right\}$ is the system of Rademacher functions. Let

$$
\begin{aligned}
c_{k}^{2} & =a_{k}^{2}+b_{k}^{2}, \quad B_{N}^{2}=\frac{1}{2} \sum_{1}^{N} c_{k}^{2} \\
S_{N} & =\sum_{1}^{N} \varphi_{k}(t) A_{k}(x),
\end{aligned}
$$

and let $\omega(p)$ be a function of $p$ increasing to $+\infty$ with $p$, such that $p / \omega(p)$ increases and that $\Sigma 1 / p \omega(p)<\infty$. Then, under the assumptions

I See Bulletin des Sciences Mathématiques, 74 (1950). 


$$
B_{N}^{2} \rightarrow \infty, c_{N}^{2}=O\left\{\frac{B_{N}^{2}}{\omega\left(B_{N}^{2}\right)}\right\}
$$

one has, for almost every value of $t$,

$$
\lim \sup \frac{S_{N}}{\left(2 B_{N}^{2} \log \log B_{N}\right)^{\frac{\mathrm{t}}{2}}}=1
$$

almost everywhere in $x$, that is to say the law of the iterated logarithm is true for almost all series (2.1.3).

The proof will be based on Theorem (1.2.1).

2. For a given $x$, the law of the iterated logarithm applied to the series (2.1.3) in which $t$ is the variable, gives

p.p. in $t$, provided

$$
\lim \sup \frac{\sum_{1}^{N} A_{n}(x) \varphi_{n}(t)}{\left\{2 \sum_{1}^{N} A_{n}^{2}(x) \log \log \sum_{1}^{N} A_{n}^{2}(x)\right\}^{\frac{1}{3}}}=1,
$$

$$
\sum_{1}^{N} A_{n}^{2}(x) \rightarrow \infty, A_{n}^{2}(x)=o\left\{\begin{array}{c}
\sum_{1}^{N} A_{n}^{2}(x) \\
\hdashline \log \log \sum_{1}^{N} A_{n}^{2}(x)
\end{array}\right\} .
$$

One has

$$
\begin{aligned}
& A_{n}(x)=a_{n} \cos n x+b_{n} \sin n x=c_{n} \cos \left(n x-\alpha_{n}\right), \\
& A_{n}^{2}(x)=\frac{1}{2} c_{n}^{2}\left\{1+\cos \left(2 n x-2 \alpha_{n}\right)\right\} .
\end{aligned}
$$

Hence, by Theorem (1.2.1) and on account of the condition $c_{n}^{2}=O\left\{B_{n}^{2} / \omega\left(B_{n}^{2}\right)\right\}$, one has

$$
B_{N}^{2} \sum_{1}^{N} A_{n}^{2}(x) \rightarrow 1 \text { p.p. in } x \text {. }
$$

On the other hand, condition (2.2.2) is satisfied p.p. in $x$, because $\Sigma 1 / p \omega(p)<\infty$ and $\omega(p)$ increasing imply $\log p / \omega(p) \rightarrow 0$. Hence

$$
c_{N}^{2}=o\left\{\frac{B_{N}^{2}}{\log \log B_{N}}\right\},
$$

which together with (2.2.3) implies (2.2.2).

Finally, (2.2.1) and (2.2.3) give

$$
\lim \sup \frac{S_{N}}{\left(2 B_{N}^{2} \log \log B_{N}\right)^{\frac{1}{2}}}=1
$$

p.p. in $t$ and $x$, and so also the theorem as stated.

17 -533807. Acta Mathematica. 91. Imprimé le 30 octobre 1954. 
3. Additional remarks. a) We do not know whether the condition

$$
c_{N}^{2}=O\left\{\frac{B_{N}^{2}}{\omega\left(B_{N}^{2}\right)}\right\}
$$

with $\sum 1 / p \omega(p)<\infty$, can be improved or not (this condition is certainly satisfied if $\left.c_{n}=O(1)\right)$. But the argument used here would break down if $\Sigma 1 / p \omega(p)$ were divergent. This follows from Theorem (1.3.1).

In the rest of this chapter the function $\omega(u)$ will be supposed to have the properties assumed in Theorem (2.1.2).

b) The following is an analogue of Theorem (2.1.2) for power series

$$
\sum_{1}^{\infty} \varphi_{n}(t) c_{n} e^{i n x},
$$

for which we set

$$
C_{N}^{2}=\sum_{1}^{N}\left|c_{k}^{2}\right|, S_{N}=\sum_{1}^{N} \varphi_{k}(t) c_{k} e^{i k x}
$$

(2.3.3) Theorem. For almost every $t$ we have

$$
\lim \sup \frac{\left|S_{N}\right|}{\left(C_{N}^{2} \log \log C_{N}\right)^{\frac{1}{t}}}=1
$$

almost everywhere in $x$, provided

$$
C_{N}^{2} \rightarrow \infty, c_{N}^{2}=O\left\{\frac{c_{N}^{2}}{\omega\left(c_{N}^{2}\right)}\right\}
$$

We note that the factor 2 is missing in the denominator in (2.3.4).

Let us set $S_{N}=U_{N}+i V_{N}$. From Theorem (2.1.2) it follows that for almost every point $(x, t)$ and every rational $\alpha$ we have

$$
\lim \sup \frac{U_{N} \cos \alpha \pi+V_{N} \sin \alpha \pi}{\left(C_{N}^{2} \log \log C_{N}\right)^{\dagger}}=1,
$$

and from this we easily deduce that (2.3.4) holds for almost every point $(x, t)$ (compare a similar argument used in Salem and Zygmund, loc, cit.).

c) Kolmogoroff's result quoted at the beginning of this chapter has an analogue for the case in which the series $\Sigma b_{k}$ converges. Writing $R_{N}=z_{N}+z_{N+1}+\cdots, \beta_{N}^{2}=$ $=b_{N}+b_{N+1}+\cdots$, one has, with probability 1 ,

$$
\lim \sup R_{N} /\left(2 \beta_{N}^{2} \log \log 1 / \beta_{N}\right)^{1}=1
$$

provided $\left|z_{N}\right| \leq o\left\{\beta_{N}^{2} / \log \log \left(1 / \beta_{N}\right)\right\}$. 
Combining this result with the proof of Theorem (1.2.1), and using Theorem (1.6.1) we get the following

(2.3.5) Theorem. Let us suppose that the sum $\Sigma\left(a_{k}^{2}+b_{k}^{2}\right)$ corresponding to the series (2.1.3) is finite, so that the series (2.1.3) converges at almost every point $(x, t),{ }^{1}$ and let us set

$$
c_{n}^{2}=a_{n}^{2}+b_{n}^{2}, \beta_{N}^{2}=\frac{1}{2} \sum_{N}^{\infty} c_{n}^{2}, R_{N}=\sum_{N}^{\infty} \varphi_{n}(t) A_{n}(x)
$$

Then, for almost every $t$, we have

$$
\lim \sup \frac{R_{N}}{\left(2 \beta_{N}^{2} \log \log 1 / \beta_{n}\right)^{\natural}}=1
$$

almost everywhere in $x$, provided

$$
c_{N}^{2}=O\left\{\frac{\beta_{N}^{2}}{\omega\left(1 / \beta_{N}^{2}\right)}\right\} .
$$

An obvious analogue holds for power series (2.3.1), with $\Sigma\left|c_{k}\right|^{2}$ finite.

\section{Chapter I I I}

\section{The Central Limit Theorem}

1. In this Section, $\omega(u), c_{N}, B_{N}, S_{N}$ have the same meaning as in Theorem (2.1.2).

(3.1.1) Theorem. Under the same conditions as in Theorem (2.1.2), namely

$$
B_{N}^{2} \rightarrow \infty, \quad c_{N}^{2}=O\left\{\frac{B_{N}^{2}}{\omega\left(B_{N}^{2}\right)}\right\},
$$

the distribution function of $S_{N} / B_{N}$ tends, for almost every $t$, to the Gaussian distribution with mean value zero and dispersion 1.

It is easily seen that the assumptions imply also

$$
\underset{1 \leq k \leq N}{\operatorname{Max}} c_{k}^{2}=O\left\{\frac{B_{N}^{2}}{\omega\left(B_{N}^{2}\right)}\right\} .
$$

We shall suppose, for the sake of brevity, that the series $\Sigma \varphi_{k}(t) A_{k}(x)$ is a purely cosine series (our proof is immediately adaptable to the general case by writing $\left.a_{n} \cos n x+b_{n} \sin n x=c_{n} \cos \left(n x-\alpha_{n}\right)\right)$, and it will be convenient to replace the variable $x$ by $2 \pi x$, so that the series becomes $\Sigma \varphi_{k}(t) a_{k} \cos 2 \pi k x$.

1 Seo Paley and Zyamund, loc. cit., or Zygmund, Trigonometrical Series, p. 125. 
For a given $t$, let $E_{N}(y)$ be the set of points $x$ of the interval $(0,1)$ at which $S_{N} / B_{N} \leq y$, and let $F_{N}(y)$ be the measure of $\boldsymbol{E}_{N}(y)$. Then $\boldsymbol{F}_{N}(y)$ is the distribution function of $S_{N} / B_{N}$. In order to prove our theorem it will be enough to prove that over every finite range of $\lambda$ the characteristic function of $F_{N}(y)$ approaches uniformly that of the Gaussian distribution, for almost every $t$. This characteristic function is

$$
\int_{-\infty}^{+\infty} e^{i \lambda y} d F_{N}(y)=\int_{0}^{1} e^{i \lambda s_{N^{\prime}}{ }^{*}} d x
$$

and we have to prove that for almost all $t$ the last integral tends to $\exp \left(-\frac{1}{2} \lambda^{2}\right)$ uniformly over any finite range of $\lambda .^{1}$

Let us now fix $t$. Since

one has

$$
e^{z}=(1+z) e^{\frac{1}{2} z^{1}+o\left(\left[z^{13}\right)\right.} \text { as } z \rightarrow 0
$$

(3.1.3) $\quad e^{i \lambda S_{N^{\prime}} B_{N}}=\prod_{k=1}^{N}\left(1+i \frac{\lambda a_{k}}{B_{N}} \varphi_{k}(t) \cos 2 \pi k x\right) \exp \left\{\left(-\frac{1}{2} \lambda^{2} \sum_{1}^{N} \frac{a_{k}^{2}}{B_{N}^{2}} \cos ^{2} 2 \pi k x\right)+o(1)\right\}$,

where the term $o(1)$ tends to 0 , uniformly in $x$, as $N \rightarrow \infty$, since $\lambda=O(1)$ and

$$
\max _{1 \leq k \leq N} a_{k}^{2}=O\left\{\frac{B_{N}^{2}}{\omega\left(B_{N}^{2}\right)}\right\}=o\left(B_{N}^{2}\right) .
$$

Observe now that

$$
\left|\prod_{k=1}^{N}\left(1+i \frac{\lambda a_{k}}{B_{N}} \varphi_{k}(t) \cos 2 \pi k x\right)\right| \leq \prod_{k=1}^{N}\left(1+\frac{\lambda^{2} a_{k}^{2}}{B_{N}^{2}}\right)^{\frac{1}{2}} \leq e^{\lambda^{2}},
$$

and that, writing

$$
\sum_{1}^{N} \frac{a_{k}^{2}}{B_{N}^{2}} \cos ^{2} 2 \pi k x=1+\sum_{1}^{N} \frac{a_{k}^{2}}{2 B_{N}^{2}} \cos 4 \pi k x=1+\xi_{N}(x)
$$

the measure of the set of points at which $\left|\xi_{N}(x)\right| \geq \delta>0$ is not greater than

$$
\delta^{2} \int_{0}^{1} \xi_{N}^{2} d x=\frac{1}{8} \delta^{-2}\left(a_{1}^{4}+\cdots+a_{N}^{4}\right) B_{N}^{4},
$$

and that the last quantity tends to 0 as $N \rightarrow \infty$.

Hence, with an error tending to zero (uniformly in $\lambda=O(1)$ ) as $N \rightarrow \infty$, the integral (3.1.2) is

$$
e^{-\frac{1}{2} \lambda^{\prime}} \int_{0}^{1} \prod_{1}^{N}\left(1+i \frac{\lambda a_{k}}{B_{N}} \varphi_{k}(t) \cos 2 \pi k x\right) d x
$$

1 Since the exponential function is continuous, the uniformity of convergence is (as is very well known) not indispensable here. 
and in order to prove our theorem we have to show that, p.p. in $t$,

$$
\lim _{N \rightarrow \infty} \int_{0}^{1} \prod_{1}^{N}\left(1+i \varepsilon_{k} \varphi_{k}(t) \cos 2 \pi k x\right) d x=1
$$

where we write $\varepsilon_{k}=\varepsilon_{k}(N)=\lambda a_{k} / B_{N}$.

2. Let us set

$$
\begin{aligned}
J_{N}(t) & =\int_{0}^{1} \prod_{1}^{N}\left(1+i \varepsilon_{k} \varphi_{k}(t) \cos 2 \pi k x\right) d x-1 \\
& =\int_{0}^{1}\left\{\prod_{1}^{N}\left(1+i \varepsilon_{k} \varphi_{k}(t) \cos 2 \pi k x\right)-1\right\} d x .
\end{aligned}
$$

Writing $\Pi(x)=\prod_{1}^{N}\left(1+i \varepsilon_{k} \varphi_{k}(t) \cos 2 \pi k x\right)$, one has

and

$$
\left|J_{N}(t)\right|^{2}=\int_{0}^{1} \int_{0}^{1}\{\Pi(x)-1\}\{\bar{\Pi}(y)-1\} d x d y
$$

Now

$$
\int_{0}^{1}\left|J_{N}(t)\right|^{2} d t=\int_{0}^{1} \int_{0}^{1} d x d y \int_{\theta}^{1}\{\Pi(x)-1\}\{\bar{\Pi}(y)-1\} d t
$$

$$
\int_{0}^{1} \Pi(x) d t=\int_{0}^{1} \Pi(y) d t=1
$$

and

$$
\begin{aligned}
\int_{0}^{1} \Pi(x) \Pi(y) d t= & \int_{0}^{1} \prod_{1}^{N}\left\{1+\varepsilon_{k}^{2} \cos 2 \pi k x \cos 2 \pi k y\right. \\
& \left.+i \varepsilon_{k}(\cos 2 \pi k x-\cos 2 \pi k y) \varphi_{k}(t)\right\} d t \\
& =\prod_{1}^{N}\left(1+\varepsilon_{k}^{2} \cos 2 \pi k x \cos 2 \pi k y\right) .
\end{aligned}
$$

Hence

$$
\begin{aligned}
\int_{0}^{1}\left|J_{N}(t)\right|^{2} d t & =\int_{0}^{1} \int_{0}^{1} d x d y\left\{\prod_{1}^{N}\left(1+\varepsilon_{k}^{2} \cos 2 \pi k x \cos 2 \pi k y\right)-1\right\} \\
& =\int_{0}^{1} \int_{0}^{1} \prod_{1}^{N}\left(1+\varepsilon_{k}^{2} \cos 2 \pi k x \cos 2 \pi k y\right) d x d y-1 \\
& <\int_{0}^{1} \int_{0}^{1}\left\{\exp \sum_{1}^{N} \varepsilon_{k}^{2} \cos 2 \pi k x \cos 2 \pi k y\right\} d x d y-1
\end{aligned}
$$


Using the fact that $e^{u}=1+u+\frac{1}{2} u^{2} e^{\eta u}, 0<\eta<1$, with

$$
u=\sum_{1}^{N} \varepsilon_{k}^{2} \cos 2 \pi k x \cos 2 \pi k y
$$

and observing that $|u| \leq \sum_{1}^{N} \varepsilon_{k}^{2}=2 \lambda^{2}$, one has, since $\int_{0}^{1} \int_{0}^{1} u d x d y=0$,

$$
\begin{aligned}
\int_{0}^{1}\left|J_{N}(t)\right|^{2} d t & \leq \frac{1}{2} e^{2 \lambda^{3}} \int_{0}^{1} \int_{0}^{1}\left(\sum_{1}^{N} \varepsilon_{k}^{2} \cos 2 \pi k x \cos 2 \pi k y\right)^{2} d x d y \\
& =\frac{1}{8} e^{2 \lambda^{2}} \sum_{1}^{N} \varepsilon_{k}^{4}=\frac{1}{8} \lambda^{4} e^{2 \lambda^{*}} \frac{\sum_{1}^{N} a_{k}^{4}}{B_{N}^{4}}
\end{aligned}
$$

and since

$$
\max _{1 \leq k \leq N} a_{k}^{2}=O\left\{\frac{B_{N}^{2}}{\omega\left(B_{N}^{2}\right)}\right\}
$$

one has

$$
\int_{0}^{1}\left|J_{N}(t)\right|^{2} d t=O\left\{\frac{1}{\omega\left(B_{N}^{2}\right)}\right\}
$$

Let us now fix a number $\theta>1$, and let $N$, be the first integer such that $\theta^{j} \leq B_{N_{j}}^{2}<\theta^{j+1}$. In Chapter $I$, in connection with the proof of Theorem (1.2.1), we showed that such an integer always exists for $j$ large enough. Thus

$$
\int_{0}^{1}\left|J_{N_{j}}(t)\right|^{2} d t=O\left\{-\frac{1}{\omega\left(\partial^{j}\right)}\right\}
$$

Since $\Sigma 1 / p \omega(p)<\infty$, we have $\sum 1 / \omega\left(\theta^{j}\right)<\infty$, and thus $J_{N_{j}}$ tends to zero for almost every $t$. We have therefore shown that

$$
\int_{0}^{1} e^{i \lambda S_{N_{j} / B} N_{j}} d x \rightarrow e^{-\frac{1}{2} \lambda \lambda^{2}}
$$

p.p. in $t$, and uniformly over any finite range of $\lambda$.

3. Let us now consider an integer $m$ such that

$$
N_{1} \leq m<N_{j+1}
$$

and let

$$
\Delta=\int_{0}^{1} e^{i \lambda S_{m} / B_{m}} d x-\int_{0}^{1} e^{i \lambda S_{N_{j} / B_{N_{j}}} d x}
$$


One has

$$
\begin{aligned}
|\Delta| & \leq \int_{0}^{1}\left|\lambda\left(\frac{S_{m}}{B_{m}}-\frac{S_{N_{j}}}{B_{N_{j}}}\right)\right| d x \\
|\Delta|^{2} & \leq \lambda^{2} \int_{0}^{1}\left[\frac{S_{m}-S_{N_{j}}}{B_{m}}+S_{N_{j}}\left(\frac{1}{B_{m}}-\frac{1}{B_{N_{j}}}\right)\right]^{2} d x \\
& \leq \frac{2 \lambda^{2}}{B_{m}^{2}} \int_{0}^{1}\left(S_{m}-S_{N_{j}}\right)^{2} d x+2 \lambda^{2} \frac{\left(B_{N_{j+1}}-B_{N_{j}}\right)^{2}}{B_{m}^{2} B_{N_{j}}^{2}} \int_{0}^{1} S_{N_{j}}^{2} d x \\
& \leq \frac{2 \lambda^{2}}{B_{N_{j}}^{2}} \int_{0}^{1}\left(S_{N_{j+1}}-S_{N_{j}}\right)^{2} d x+2 \lambda^{2} \frac{\left(B_{N_{j+1}}-B_{N_{j}}\right)^{2}}{B_{N_{j}}^{4}} \int_{0}^{1} S_{N_{j}}^{2} d x \\
& =2 \lambda^{2}-\frac{B_{N_{j+1}}^{2}-B_{N_{j}}^{2}}{B_{N_{j}}^{2}}+2 \lambda^{2} \frac{\left(B_{N_{j+1}}-B_{N_{j}}\right)^{2}}{B_{N_{j}}^{2}} \\
& \leq 4 \lambda^{2} \frac{B_{N_{j+1}}^{2}-B_{N_{j}}^{2}}{B_{N_{j}}^{2}} \leq 4 \lambda^{2} \frac{\theta^{j+2}-\theta^{\prime}}{\theta^{j}}=4 \lambda^{2}\left(\theta^{2}-1\right) .
\end{aligned}
$$

Hence

$$
\int_{0}^{1} e^{i \lambda S_{m} / B_{m}} d x=\int_{0}^{1} e^{i \lambda S_{N_{j} / B_{N}}} d x+\Delta
$$

where

$$
|\Delta| \leq 2|\lambda|\left(\theta^{2}-1\right)^{l} \text { and } \lim \int_{0}^{1} e^{i \lambda S_{N_{j} / B_{N}}} d x=e^{-\frac{1}{2} \lambda^{2}}
$$

Since $\theta$ can be taken arbitrarily close to 1 , this proves that

$$
\int_{0}^{1} e^{t \lambda s_{m} / B_{m}} d x \rightarrow e^{-\frac{1}{2} \lambda^{2}} \text { p.p. in } t
$$

uniformly over any finite range of $\lambda$, and this completes the proof of Theorem (3.1.1).

Whether the condition $c_{N}^{2}=O\left\{B_{N}^{2} / \omega\left(B_{N}^{2}\right)\right\}$ is the best possible one, we are not able to decide.

4. The result that follows is a generalization of Theorem (3.1.1).

(3.4.1) Theorem. The notation being the same and under the same conditions as in Theorem (3.1.1), the distribution function of $S_{N} / B_{N}$ on every fixed set $G$ of positive measure tends to the Gaussian distribution, for all values of $t$ with the possible exception 
of a set of measure zero which is independent of the set $G$. More precisely, $E_{N}(y)$ being the set of points $x$ in $(0,1)$ such that $S_{N} / B_{N} \leq y$, and

$$
F_{N}(y, G)=\frac{\operatorname{meas}\left[E_{N}(y) \cdot G\right]}{\operatorname{meas} G}
$$

$F_{N}(y, G)$ tends to the Gaussian distribution with mean value zero and dispersion 1.

We have

$$
\int_{-\infty}^{+\infty} e^{i \lambda y} d F_{N}(y, G)=|G|^{-1} \int_{G} e^{i \lambda s_{N^{\prime}}{ }^{*}} d x
$$

where $|G|$ denotes the measure of $G$, and we have to prove that the last expression tends to $e^{\frac{3}{2} \lambda^{2}}$, uniformly over any finite range of $\lambda$, for all values of $t$ except in a set $H_{t}$ of measure zero, $H_{t}$ being independent of $G$.

Our theorem will be established if we prove it in the case when $G$ is an interval with rational end points. For then it would be proved when $G$ is a sum of a finite set of intervals $I$, whence we would get the result when $G$ is the most general open set in $(0,1)$. Since every measurable set is contained in an open set of measure differing as little as we please, we would obtain the result in the general case.

Without loss of generality, we may assume that $I$ is an interval of the form $(0, \alpha)$, where $\alpha$ is rational, $0 \cdots \alpha=1$. Suppose now we can prove that for a given $\alpha$,

$$
\alpha^{1} \int_{0}^{x} e^{i \lambda S_{N^{\prime}} N} d x \rightarrow e^{\frac{1}{2} \lambda^{2}}
$$

almost everywhere in $t$, that is to say with the exception of a set $H_{t}(\alpha)$ of measure zero. Our result will then follow, since the set $H_{t}=\sum_{\alpha} H_{t}(\alpha)$, summation being extended over all rational numbers $\alpha$, is also of measure zero.

Thus we have to prove that $(3.4 .2)$ is, for a given $\alpha$, true p.p. in $t$. As in the proof of Theorem (3.1.1), it is enough to show that

$$
K_{N}(t)=\int_{0}^{\alpha} \prod_{1}^{N}\left(1+i \varepsilon_{k} \varphi_{k}(t) \cos 2 \pi k x\right) d x-\alpha
$$

tends to zero p.p. in $t$, where $\varepsilon_{k}=\lambda a_{k} / B_{N}$ and $N \rightarrow \infty$.

The proof proceeds exactly in the same way as before until we get

$$
\int_{0}^{1}\left|K_{N}(l)\right|^{2} d t=\int_{0}^{\alpha} \int_{0}^{\alpha} e^{\sum_{1}^{2} \varepsilon_{k}^{2} \cos 2 \pi k x \cos 2 \pi k y} d x d y-\alpha^{2}
$$


Writing now again $e^{u}=-1+u+\frac{1}{2} u^{2} e^{\eta u}, 0<\eta<1$, we observe that

$$
\int_{0}^{\alpha} \int_{0}^{\alpha} \varepsilon_{k}^{2} \cos 2 \pi k x \cos 2 \pi k y d x d y=\frac{\varepsilon_{k}^{2} \sin ^{2} 2 \pi k \alpha}{4 \pi^{2} k^{2}}<\frac{\varepsilon_{k}^{2}}{k^{2}}
$$

and thus

$$
\left|\int_{0}^{\alpha} \int_{0}^{\alpha} u d x d y\right| \leq 2 \frac{\lambda^{2}}{B_{N}^{2}} \max _{1 \leq k \leq N} a_{k}^{2}=O\left\{\frac{1}{\omega\left(B_{N}^{2}\right)}\right\}
$$

Noting also that

$$
\int_{0}^{\alpha} \int_{0}^{\alpha} u^{2} d x d y \leq \int_{0}^{1} \int_{0}^{1} u^{2} d x d y
$$

we get

$$
\int_{0}^{1}\left|K_{N}(t)\right|^{2} d t=O\left\{1 / \omega\left(B_{N}^{2}\right)\right\}
$$

from which place the proof proceeds as before.

5. Theorems (3.1.1) and (3.4.1) have analogues for power series

$$
\sum_{1}^{\infty} c_{k} e^{2 \pi i k x} \varphi_{k}(t)
$$

whose partial sums we shall again denote by $S_{N}(x)$.

(3.5.2) Theorem. If

$$
C_{N}^{2}=\frac{1}{2} \sum_{1}^{N}\left|c_{k}\right|^{2}, c_{N}^{2}=O\left\{C_{N}^{2} / \omega\left(C_{N}^{2}\right)\right\}
$$

then the two-dimensional distribution function of $S_{N}(x) / C_{N}$ tends, for almost every $t$, to the Gaussian distribution

$$
\frac{1}{2 \pi} \int_{-\infty}^{\xi} \int_{-\infty}^{\eta} e^{\cdot \frac{1}{2}\left(\lambda^{2}+\mu^{2}\right)} d \lambda d \mu
$$

It is enough to sketch the proof. ${ }^{1}$ Let $c_{k}=\left|c_{k}\right| e^{i \alpha_{k}}$, and let $U_{N}$ and $V_{N}$ denote, respectively, the real and imaginary parts of $S_{N}$. Let $\boldsymbol{F}_{N}(\xi, \eta)$ denote the measure of the set of points $x, 0 \leq x<1$, such that $U_{N}(x) / C_{N} \leq \xi, V_{N}(x) / C_{N} \leq \eta$, simultaneously. The characteristic function of $F_{N}$ is

1 See also the authors' notes "On lacunary trigonometric series" part I, Proc. Nat. Acad., 33 (1947), pp. 333-338, esp. p. 337, and part II, Ibid. 34 (1948), pp. 54-62. 


$$
\begin{aligned}
& \int_{-\infty}^{+\infty} \int_{-\infty}^{+\infty} e^{i(\lambda \xi+\mu \eta)} d F_{N}(\xi, \eta)=\int_{0}^{1} e^{i\left(\lambda U_{N}+\mu V_{N}\right) / C_{N}} d x \\
& =\int_{0}^{1} \exp i C_{N}^{-1}\left\{\sum_{1}^{N}\left|c_{k}\right|\left[\lambda \cos \left(2 \pi k x+\alpha_{k}\right)+\mu \sin \left(2 \pi k x+\alpha_{k}\right)\right] \varphi_{k}(t)\right\} d x \\
& =\int_{0}^{1} \exp \left\{i C_{N}^{-1}\left(\lambda^{2}+\mu^{2}\right)^{\frac{1}{2}} \sum_{1}^{N}\left|c_{k}\right| \cos \left(2 \pi k x+\alpha_{k}^{\prime}\right) \varphi_{k}(t)\right\} d x,
\end{aligned}
$$

where the $\alpha_{k}^{\prime}$ now also depend on $\lambda$ and $\mu$.

To the last integrand we apply a formula analogous to (3.1.3) and we find that for $\lambda^{2}+\mu^{2}=O(1)$ our integral is

$$
\left.e^{-\frac{1}{2}\left(\lambda^{2}+\mu^{2}\right)} \int_{0}^{1} \prod_{1}^{N}\left\{1+i\left|c_{k}\right|\left(\lambda^{2}+\mu^{2}\right)^{\frac{1}{2}} C_{N}^{-1} \varphi_{k}(t) \cos 2 \pi k x+\alpha_{k}^{\prime}\right)\right\} d x
$$

with an error tending uniformly to zero. The second factor here tends to 1 p.p. in $t$, since after an obvious change of notation it reduces to the integral in (3.1.4), provided in the latter we replace $\cos 2 \pi k x$ by $\cos \left(2 \pi k x+\alpha_{k}^{\prime}\right)$, which does not affect the validity of (3.1.4). Hence, p.p. in $t$, the characteristic function of $F_{N}(\xi, \eta)$ tends to $e^{-\frac{1}{2}\left(\lambda^{2}+\mu^{2}\right)}$, which completes the proof of Theorem (3.5.1).

It is clear that the conclusion of the theorem holds if we consider the distribution function of $S_{N}(x) / C_{N}$ over any set of positive measure in the interval $0 \leq x<1$.

This result and Theorem (3.4.2) have analogues in the case when the series are of the class $L^{2}$, i.e. when the sum of the squares of the moduli of the coefficients of the series is finite. Then, instead of the normalized partial sums we consider the normalized remainders of the series and show that, under condition (2.3.6), the distribution functions of these expressions tend, p.p. in $t$, to the Gaussian distribution. The proofs remain unchanged.

6. So far we have been considering only the partial sums or remainders of series. One can easily extend the results to general methods of summability (see, for example, the authors' note cited in the preceding Section, where this is done for lacunary series). We shall, however, confine our attention to the Abel-Poisson method, which is interesting in view of its function-theoretic aspect.

(3.6.1) Theorem. Suppose that $\Sigma\left(a_{k}^{2}+b_{k}^{2}\right)=\infty$, and let

$$
c_{k}^{2}=a_{k}^{2}+b_{k}^{2}, B^{2}(r)=\frac{1}{2} \Sigma c_{k}^{2} r^{2 k}, 0 \leq r<1
$$


Then, as $r \rightarrow 1$, the distribution function of

$$
f_{r}(x)=\Sigma\left(a_{k} \cos 2 \pi k x+b_{k} \sin 2 \pi k x\right) \varphi_{k}(t) r^{k}
$$

tends, p.p. in $t$, to the Gaussian distribution with mean value zero and dispersion 1 , provided

$$
\max _{1 \leq k<\infty}\left(c_{k}^{2} r^{2 k}\right)=O\left\{B^{2}(r) / \omega\left(B^{2}(r)\right)\right\}
$$

in particular, provided $c_{k}=O(1)$.

The proof is the same as that of Theorem (3.1.1). Extensions to power series, sets of positive measure and series of the class $L^{2}$ are straightforward.

\section{ChAPTER I V}

\section{On the Maximum of Trigonometric Polynomials whose Coefficients have Random Signs}

1. In this chapter we shall consider series of the form

$$
\sum_{1}^{\infty} r_{m} \varphi_{m}(t) \cos m x
$$

where $\left\{\varphi_{m}(t)\right\}$ is the Rademacher system, and where we consider purely cosine series only to simplify writing, there being no difficulty in extending the results to the series of the form $\sum r_{m} \varphi_{m}(t) \cos \left(m x-\alpha_{m}\right)$.

Writing

$$
P_{n}=P_{n}(x, t)=\sum_{1}^{n} r_{m} \varphi_{m}(t) \cos m x
$$

we consider

$$
M_{n}=M_{n}(t)=\max _{x}\left|P_{n}(x, t)\right|
$$

and our main problem will be to find, under fairly general conditions, the order of magnitude of $M_{n}$ for almost every $t$; more exactly, to determine a function of $n$, say, $\Omega(n)$, such that

$$
c \leq \lim \text { inf } \frac{M_{n}(t)}{\Omega(n)} \leq \lim \sup \frac{M_{n}(t)}{\Omega(n)} \leq C
$$

almost everywhere in $t, c>0$ and $C$ being constants.

Analogous results will be given for power series of the form

$$
\sum r_{m} e^{i\left(m x+2 \pi \alpha_{m}\right)}
$$


where the phases $\alpha_{m}$ are variable. The Rademacher functions are replaced here by the Steinhaus functions $e^{2 \pi i \alpha_{m}}$, which are functions of a single variable $t, 0 \leq t \leq 1$, and are obtained from mapping this interval onto the unit cube $0 \leq \alpha_{m} \leq 1$ of in. finitely many dimensions (see Steinhaus, Studia Math., 2 (1930), pp. 21-40).

\section{Part 1. Rademacher Functions}

2. We begin by proving a number of lemmas which we shall need later on.

(4.2.1) Lemma. Let $f_{n}=\sum_{1}^{n} c_{m} \varphi_{m}(t)$, where $\left\{\varphi_{m}\right\}$ is the Rademacher system and the $c_{m}$ are real constants. Let $C_{n}=\sum_{1}^{n} c_{m}^{2}, D_{n}=\sum_{1}^{n} c_{m}^{4}$ and let $\lambda$ be any real number. Then

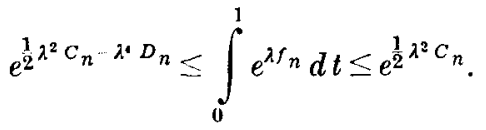

Proof. The $\varphi_{m}$ being independent functions,

$$
\begin{aligned}
\int_{0}^{1} e^{\lambda f_{n}} d t=\prod_{1}^{n} \int_{0}^{1} e^{\lambda c_{m} m_{m}} d t & =\prod_{1}^{n} e^{\lambda c_{m}+e^{\lambda c_{m}}} \\
2 & =\prod_{1}^{n}\left(1+\frac{\lambda^{2} c_{m}^{2}}{2 !}+\frac{\lambda^{4} c_{m}^{4}}{4 !}+\cdots\right) .
\end{aligned}
$$

Since $(2 p) ! \geq 2^{p} p !$, one has

$$
\int_{0}^{1} e^{\lambda f_{n}} d t \leq \prod_{m=1}^{n}\left(\sum_{p=0}^{\infty} \frac{\lambda^{2 p} c_{m}^{2 p}}{2^{p} p !}\right)=\prod_{1}^{n} e^{\frac{1}{2} \lambda^{2} c_{m}^{2}}=e^{\frac{1}{2} \lambda^{2} C_{n}}
$$

In the opposite direction,

$$
\int_{0}^{1} e^{\lambda f_{n}} d t \geq \prod_{1}^{n}\left(1+\frac{\lambda^{2} c_{m}^{2}}{2}\right)
$$

Using the fact that for $u>0$ one has $1+u>e^{u \cdot \frac{1}{2} u^{2}}$, one gets

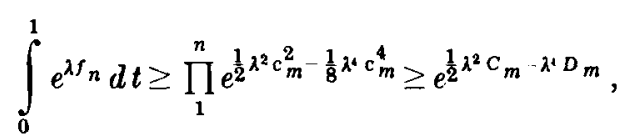

which completes the proof. ${ }^{1}$

1 Later on we shall need the lemma in the case when $n=\infty$ and $\Sigma c_{m}^{2}<\infty$. It is clear that the inequalities of the lemma hold in this case, since $\Sigma_{c_{m}} \varphi_{m}$ converges almost everywhere. 
The lemma which follows is well known but we state it in order to avoid constant repetitions. It is stated for functions of a single variable but it clearly holds for functions of several variables.

(4.2.2) Lemma. Let $g(x), a \leq x \leq b$, be a bounded real function. Suppose that

$$
|g(x)| \leq A, \quad(b-a)^{-1} \int_{a}^{b} g^{2}(x) d x=B .
$$

Then, for any positive number $\mu$,

$$
(b-a)^{-1} \int_{a}^{b} e^{\mu g(x)} d x \leq 1+\mu \sqrt{B}+\frac{B}{A^{2}} e^{\mu A} .
$$

In the case when $\int_{a}^{b} g(x) d x=0$, this inequality can be replaced by

$$
(b-a)^{-1} \int_{a}^{b} e^{\mu g} d x \leq 1+\frac{B}{A^{2}} e^{\mu A} .
$$

Proof. One has

$$
e^{\mu g}=1+\frac{\mu g}{11}+\cdots+\frac{\mu^{p} g^{p}}{p !}+\cdots
$$

Now

$$
(b-a)^{1} \int_{a}^{b}|g| d x \leq \sqrt{B}, \quad(b-a)^{1} \int_{a}^{b}|g|^{p} d x \leq A^{p-2} B=\frac{B}{A^{2}} A^{p} .
$$

Hence

$$
(b-a)^{1} \int_{a}^{b} e^{\mu g} d x \leq 1+\mu \sqrt{B}+B A^{-2} \sum_{2}^{\infty} \frac{\mu^{p} A^{p}}{p !} \leq 1+\mu \sqrt{B}+B A^{2} e^{\mu A}
$$

The second inequality, if $g(x)$ has mean value zero, is obvious.

(4.2.3) Lemma. Let $x$ be real and $P(x)=\sum_{0}^{n}\left(\alpha_{m} \cos m x+\beta_{m} \sin m x\right)$ be a trigonometric polynomial of order $n$, with real or imaginary coefficients. Let $M$ denote the maximum of $|P|$ and let 0 be a positive number less than 1 . There exists then an interval of length not less than $(1-\theta) / n$ in which $|P| \geq \theta M$.

Proof. Let $x_{0}$ be a point at which $|P|=M$, and let $x_{1}$ be the first point to the right of $x_{0}$ at which $|P|=\theta M$ (if such a point does not exist there is nothing to prove). One has 


$$
\begin{aligned}
M(\mathrm{I}-\theta) & =\left|P\left(x_{0}\right)\right|-\left|P\left(x_{1}\right)\right| \\
& \leq\left|P\left(x_{0}\right)-P\left(x_{1}\right)\right| \leq\left(x_{1}-x_{0}\right) . \operatorname{Max}\left|P^{\prime}\right| .
\end{aligned}
$$

But, by Bernstein's theorem, $\max \left|P^{\prime}\right| \leq n M$. Hence

$$
x_{1}-x_{0} \geq(1-\theta) / n,
$$

as stated.

(4.2.4) Lemma. Let $\varphi(x) \geq 0$, and suppose that

$$
\int_{0}^{1} \varphi d x \geq A>0, \quad \int_{0}^{1} \varphi^{2} d x \leq B
$$

(clearly, $A^{2} \leq B$ ). Let $\delta$ be a pasitive number less than 1 and let $|E|$ denote the measure of the set $E$ in which $\varphi \geq \delta A$. Then $|E| \geq(1-\delta)^{2} \frac{A^{2}}{B}$.

If $C E$ denotes the set complementary to $E$, then $\int_{C E} \varphi d x \leq \delta A$ and

$$
\int_{E} \varphi d x=\int_{0}^{1} \varphi d x-\int_{C E} \varphi d x \geq A(1-\delta) .
$$

But

so that

$$
\begin{gathered}
\int_{E} \varphi d x \leq|E|^{\frac{1}{2}}\left(\int_{0}^{1} \varphi^{2} d x\right)^{t} \leq|E|^{\dagger} B^{t} \\
A(1-\delta) \leq|E|^{\dagger} B^{\dot{1}}
\end{gathered}
$$

$$
|E| \geq(1-\delta)^{2} \frac{A^{2}}{B}
$$

(4.2.5) Lemma. Let $f_{k}=\sum_{1}^{k} c_{m} \varphi_{m}(t)$, where $\left\{\varphi_{m}\right\}$ is the Rademacher system and the $c_{m}$ are real constants. Let $n(t)$ be a measurable function taking only positive integral values and suppose that $1 \leq n(t) \leq n$. Write $C_{n}=\sum_{1}^{n} c_{m}^{2}$ and denote by $\lambda$ a positive number. Then

$$
\int_{0}^{1} e^{\lambda\left|f_{n(t)}(t)\right|} d t \leq 16 e^{\frac{1}{2} \lambda^{2} C_{n}}
$$

The proof of this well-known result is included for the convenience of the reader. 
Proof. Let $k$ be an integer, $1 \leq k \leq n$. Then, if $(\alpha, \beta)$ is any dyadic interval of length $2^{-k}\left(\alpha=p 2^{-k}, \beta=(p+1) 2^{-k}, p\right.$ an integer $)$, one has

$$
f_{k}(t)=(\beta-a)^{-1} \int_{\alpha}^{\beta} f_{n}(u) d u, \quad \alpha \leq t \leq \beta .
$$

Thus, for all $t$,

$$
\left|f_{k}(t)\right| \leq(\beta-\alpha)^{-1} \int_{\alpha}^{g}\left|f_{n}(u)\right| d u \leq \sup _{\theta} \frac{1}{\theta-t} \int_{i}^{\theta}\left|f_{n}(u)\right| d u \quad(0 \leq \theta \leq 1) .
$$

Denoting the last member of the inequality by $f_{n}^{*}(t)$, we see that

$$
\left|f_{n(t)}(t)\right| \leq f_{n}^{*}(t)
$$

By the well known inequality of Hardy and Littlewood,

$$
\int_{0}^{1}\left[f_{n}^{*}(t)\right]^{a} d t \leq 2\left(\frac{q}{q-1}\right)^{Q} \int_{0}^{1}\left|f_{n}(t)\right|^{Q} d t \quad(q>1) .
$$

Hence, if $q \geq 2$,

$$
\int_{0}^{1}\left|f_{n(t)}(t)\right|^{a} d t \leq 8 \int_{0}^{1}\left|f_{n}(t)\right|^{a} d t
$$

Now,

$$
\begin{aligned}
\int_{0}^{1} e^{\lambda\left|f_{n(t)}(t)\right|} d t & =\int_{0}^{1}\left\{e^{\lambda f_{n(t)}(t)}+e^{-\lambda f_{n(t)}(t)}\right\} d t \\
& =2 \int_{0}^{1} \sum_{0}^{\infty} \frac{\lambda^{2 \mathfrak{D}}\left[f_{n(t)}(t)\right]^{2 \mathfrak{D}}}{(2 p) !} d t \\
& \leq 16 \int_{0}^{1} \sum_{0}^{\infty} \frac{\lambda^{2 p}\left[f_{n}(t)\right]^{2 \mathfrak{p}}}{(2 p) !} d t \\
& =8 \int_{0}^{1} e^{\lambda f_{n}(t)} d t+8 \int_{0}^{1} e^{-\lambda f_{n}(t)} d t \\
& \leq 16 e^{\frac{1}{2} \lambda^{2} C_{n},}
\end{aligned}
$$

by an application of Lemma (4.2.1). 
3. We now pass to the proof of our first theorem.

(4.3.1) Theorem. Consider the series (4.1.1), denote by $\boldsymbol{P}_{n}=\boldsymbol{P}_{n}(x, t)$ the polynomial $\sum_{1}^{n} r_{m} \varphi_{m}(t) \cos m x$, and write $M_{n}=M_{n}(t)=\max _{x}\left|P_{n}(x, t)\right|, R_{n}=\sum_{1}^{n} r_{m}^{2}$. There exists an absolute constant $A$ such that

$$
\limsup _{n=\infty} \frac{M_{n}(t)}{\sqrt{R_{n} \log n}} \leq A,
$$

almost everywhere in $t$.

Proof. One has, by Lemma $(4.2 .1)^{1}$,

$$
\int_{0}^{1} e^{\lambda P_{n}} d t \leq e^{\frac{1}{2} \lambda^{2} \sum_{1}^{n} r_{m}^{2} \cos ^{2} m x} \leq e^{\frac{1}{2} \lambda^{2} R_{n}}
$$

so that

$$
\int_{0}^{1} e^{\lambda\left|P_{n}\right|} d t \leq \int_{0}^{1}\left(e^{\lambda P_{n}}+e^{-\lambda P_{n}}\right) d t \leq 2 e^{\frac{1}{2} \lambda^{2} R_{n}}
$$

Hence

$$
\int_{0}^{1} d t \int_{0}^{2 \pi} e^{\lambda\left|P_{n}\right|} d x \leq 4 \pi e^{\frac{1}{2} \lambda^{2} R_{n}}
$$

By Lemma (4.2.1), denoting by 0 a fixed positive number less than 1 , one has

Hence

$$
\int_{0}^{2 \pi} e^{\lambda\left|P_{n}\right|} d x>\frac{1-\theta}{n} e^{\theta \lambda M_{n}}
$$

$$
\int_{0}^{1} e^{\theta \lambda M_{n}(t)} d t<\frac{4 \pi n}{1-\theta} e^{\frac{1}{2} \lambda^{2} R_{n}}=\frac{4 \pi}{1-\theta} e^{\frac{1}{2} \lambda^{2} R_{n}+\log n} .
$$

Take $\lambda=\left(\beta R_{n}^{1} \log n\right)^{t}, \beta$ being a positive constant to be determined later on. We get

$$
\int_{0}^{1} e^{\theta \lambda M_{n}(t)} d t<\frac{4 \pi}{1-\theta} e^{\left(\frac{1}{2} \beta+1\right) \log n}
$$

Since, at present, we are not concerned with improving the value of the constant $A$, we shall now use rather crude estimates. We have

1 In what follows $\lambda$ is always positive. 


$$
\int_{0}^{1} e^{\theta \lambda M_{n}-\left(\frac{1}{2} \beta+2+\eta\right) \log n} d t<\frac{4 \pi}{1-\theta} e^{-(1+\eta) \log n},
$$

$\eta$ being a positive number. Hence

$$
\sum_{1}^{\infty} e^{\theta \lambda M_{n}-\left(\frac{1}{2} \beta+2+\eta\right) \log n}<\infty
$$

for almost all $t$. Hence, for $n \geq n_{0}=n_{0}(t)$,

$$
\theta \lambda M_{n}<\left(\frac{1}{2} \beta+2+\eta\right) \log n
$$

and, replacing $\lambda$ by its value,

$$
M_{n}<\theta^{-1} \beta^{-\frac{1}{2}}\left(\frac{1}{2} \beta+2+\eta\right)\left(R_{n} \log n\right)^{k} .
$$

This means that, for almost all $t$,

$$
\lim \sup \frac{M_{n}(t)}{\left(R_{n} \log n\right)^{\frac{1}{2}}} \leq \frac{\frac{1}{2} \beta+2+\eta}{\theta \beta^{\frac{1}{2}}} .
$$

Since $\theta$ is arbitrarily close to 1 , and $\eta$ as small as we please,

$$
\lim \sup \frac{M_{n}(t)}{\left(R_{n} \log n\right)^{\frac{1}{t}}} \leq \frac{\beta^{t}}{2}+\frac{2}{\beta^{t}}
$$

Taking now $\beta=4$,

$$
\lim \sup \frac{M_{n}(t)}{\left(R_{n} \log n\right)^{t}} \leq 2
$$

which proves the theorem and shows that the best value of $A$ is $\leq 2$. We shall show later on (see Theorem (4.6.1) below) that under certain conditions the best value of $A$ is $\leq 1$.

Remarks on Theorem (4.3.1). As we shall see later, the order of magnitude obtained for $M_{n}$ in Theorem (4.3.1) is not always the best possible one, and additional hypotheses will be required to prove the first inequality (4.1.2) with

$$
\Omega(n)=\left(R_{n} \log n\right)^{\dagger} .
$$

An almost immediate corollary of (4.3.2) is a known result (see Paley and Zygmund, loc. cit.). If $\Sigma r_{m}^{2}$ is finite, then for almost all $t$ the partial sums

$$
s_{n}=\sum_{1}^{n} r_{m} \varphi_{m}(t) \cos m x
$$

18- 633807. Acta Mathematica. 91. Imprimé le 1 novembre 1954. 
of the series (4.1.1) are $o(\log n)^{\frac{1}{2}}$, uniformly in $x$. That these partial sums are $O(\log n)^{\frac{1}{2}}$ is obvious. By dropping the first few terms of the series (4.1.1) so as to make the $R_{n}$ uniformly small, we improve the ' $O$ ' to ' $O$ '.

We shall see later on (Section 8) that more precise information can be obtained about the order of $s_{n}$ for some particular series with $\Sigma r_{m}^{2}<\infty$.

4. In order to get further results we must now prove another lemma.

(4.4.1) Lemma. Let us again consider the series (4.1.1) and keep the notation of Theorem (4.3.1). Let $\left\{n_{j}\right\}$ be an increasing sequence of positive integers and let $\mathfrak{R}_{j}^{*}=\mathfrak{M}_{j}^{*}(t)$ be the maximum, with respect to $n$, when $n_{j}<n \leq n_{j+1}$ of

$$
H_{n}(t)=\max _{x}\left|P_{n}(x, t)-P_{n_{j}}(x, t)\right| .
$$

Then, for almost all $t$,

$$
\lim \sup _{j} \frac{\mathfrak{M}_{j}^{*}(t)}{\left\{\left(R_{n_{j+1}}-R_{n_{j}}\right) \log n_{j+1}\right\}^{\frac{1}{2}}} \leq 2
$$

Proof. Let $n(t)$ be any measurable function of $t$ taking integral values only and such that $n_{j} \cdots n(t) \leq n_{j, 1}$. By Lemma (4.2.5),

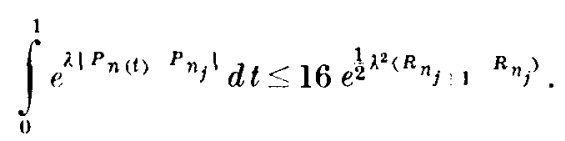

Hence

$$
\int_{0}^{1} d t \int_{0}^{2 \pi} e^{\lambda\left|P_{n(t)} P_{n_{j}}\right|} d x \leq 32 \pi e^{\frac{1}{\left.2^{\lambda^{2}\left(R_{n_{j+1}}\right.} R_{n_{j}}\right)} .}
$$

By Lemma (4.2.3), we have, with $0<\theta<1$,

$$
\int_{0}^{2 \pi} e^{\lambda \mid P_{n(t)} P_{n_{j} \mid}} d x>\frac{1-\theta}{n(t)} e^{\theta \lambda H_{n(t)}(t)} \geq \frac{1-\theta}{n_{j+1}} e^{\theta \lambda H_{n(t)}(t)} .
$$

Hence

$$
\int_{0}^{1} e^{\theta \lambda H_{n(t)}} d t<\frac{n_{j+1}}{1-\theta} 32 \pi e^{\frac{1}{2} 2^{2(}\left(R_{n_{j+1}}-R_{n_{j}}\right)},
$$

and so

$$
\int_{0}^{1} e^{\theta \lambda \geqq M_{j}^{*}(t)} d t \leq \frac{32 \pi}{1-\theta} e^{\frac{1}{2} \lambda^{2}\left(R_{n_{j+1}}-R_{n_{j}}\right)+\log n_{j+1}} .
$$


Les us take, as in the proof of Theorem (4.2.1), $\lambda=2\left(\frac{\log n_{j+1}}{R_{n_{j+1}}-R_{n_{j}}}\right)^{\frac{1}{2}}$ and let $\eta>0$. Then

$$
\int_{0}^{1} e^{\theta \lambda M M_{j}^{*}(t)(4: \eta) \log n_{j+1}} d t \leq \frac{32 \pi}{1-\theta} e^{-(1+\eta) \log n_{j+1}}
$$

and so, exactly as in the proof of Theorem (4.2.1),

$$
\lim \sup _{j} \frac{\mathfrak{M}_{j}^{*}(t)}{\left\{\left(R_{n_{j i 1}}-R_{n_{j}}\right) \log n_{j+1}\right\}^{\frac{1}{2}}} \leq \frac{4+\eta}{2 \bar{\theta}}
$$

and the lemma follows by taking $\eta$ arbitrarily small and $\theta$ arbitrarily close to 1 .

5. We proceed now to prove, under certain conditions, the first part of in. equality (4.1.2), with $\Omega(n)=\left(R_{n} \log n\right)^{\frac{1}{t}}$.

(4.5.1) Theorem. Let us consider again the series (4.1.1), the expressions $P_{n}, M_{n}$, $R_{n}$ having the same meaning as in Theorem (4.2.1). Let $T_{n}==\sum_{1}^{n} r_{m}^{4}$.

We make the following assumptions:

a) $T_{n} / R_{n}^{2}=O\left(n^{\gamma}\right)$ for some positive $\gamma$ (clearly, $\left.\gamma \leq 1\right)$, this assumption obviously implying that $\Sigma r_{m}^{2}=\infty$.

b) $R_{n_{1}} / R_{n_{2}} \rightarrow \mathrm{l}$ if $n_{1}$ and $n_{2}$ increase indefinitely in such a way that $n_{1} / n_{2} \rightarrow 1$.

Under these assumptions, one has, almost everywhere in $t$,

$$
\lim \inf _{n} \frac{M_{n}(t)}{\left(R_{n} \log n\right)^{\frac{1}{t}}} \geq c(\gamma),
$$

$c(\gamma)$ being a positive constant depending on $\gamma$ only.

Proof. Let us set $I_{n}(t)=\frac{1}{2 \pi} \int_{0}^{2 \pi} e^{\lambda P_{n}} d x$, so that, by Lemma (4.2.1),

$$
\begin{aligned}
\int_{0}^{1} I_{n}(t) d t & =\frac{1}{2 \pi} \int_{0}^{2 \pi} d x \int_{0}^{1} e^{\lambda P_{n}} d t \geq \frac{1}{2 \pi} \int_{0}^{2 \pi} e^{\frac{1}{2} \lambda^{2} \sum_{0}^{n} r_{m}^{2} \cos ^{2} m x-\lambda^{4} T_{n}} d x \\
& =e^{\frac{1}{4} \lambda^{2} R_{n}-\lambda^{2} T_{n}} \frac{1}{2 \pi} \int_{0}^{2 \pi} e^{\frac{1}{4} \lambda^{2} \sum_{1}^{n} r_{m}^{2} \cos 2 m x} d x
\end{aligned}
$$


Now, since

$$
e^{\frac{1}{4} \lambda^{2} \sum_{1}^{n} r_{m}^{2} \cos 2 m x}>1+\frac{1}{4} \lambda^{2} \sum_{1}^{n} r_{m}^{2} \cos 2 m x
$$

the mean value of the exponential exceeds 1 , so that

$$
\int_{0}^{1} I_{n}(t) d t>e^{\frac{1}{4} \lambda^{2} R_{n}-\lambda^{c} T_{n}}
$$

We proceed now to obtain an inequality in the opposite direction for $\int_{0}^{1} l_{n}^{2}(t) d t$, which, together with (4.5.2), will enable us to apply the result of Lemma (4.2.4).

We have

$$
I_{n}^{2}(t)=\frac{1}{4 \pi^{2}} \int_{0}^{2 \pi} \int_{0}^{2 \pi} e^{\lambda\left(P_{n}(x, t)+P_{n}(y, t)\right]} d x d y
$$

so that

$$
\int_{0}^{1} I_{n}^{2}(t) d t \leq \frac{1}{4 \pi^{2}} \int_{0}^{2 \pi} \int_{0}^{2 \pi} d x d y \int_{0}^{1} e^{\lambda\left[P_{n}(x, t)+P_{n}(y, t)\right]} d t
$$

Thus, by Lemma (4.2.1),

Writing

$$
\int_{0}^{1} I_{n}^{2}(t) d t \leq \frac{1}{4 \pi^{2}} \int_{0}^{2 \pi} \int_{0}^{2 \pi} e^{\frac{1}{2} \lambda^{2} \sum_{1}^{n} r_{m}^{2}(\cos m x+\cos m y)^{2}} d x d y
$$

$$
(\cos m x+\cos m y)^{2}=1+\frac{1}{2} \cos 2 m x+\frac{1}{2} \cos 2 m y+2 \cos m x \cos m y
$$

one has, putting

$$
S_{n}=S_{n}(x, y)=\sum_{1}^{n}\left\{\frac{1}{2} r_{m}^{2} \cos 2 m x+\frac{1}{2} r_{m}^{2} \cos 2 m y+2 r_{m}^{2} \cos m x \cos m y_{\}}^{\}},\right.
$$

the inequality

$$
\int_{0}^{1} I_{n}^{2}(t) d t \leq e^{\frac{1}{2} \lambda^{2} R_{n}} \cdot \frac{1}{4 \pi^{2}} \int_{0}^{2 \pi} \int_{0}^{2 \pi} e^{\frac{1}{2} \lambda^{2} S_{n}} d x d y
$$

We now use Lemma (4.2.2) for the function $S_{n}(x, y)$ of two variables. We observe that $\int_{0}^{2 \pi} \int_{0}^{2 \pi} S_{n} d x d y=0$ and that the system of $3 n$ functions $\cos 2 m x, \cos 2 m y$, $\cos m x \cos m y(m=1,2, \ldots, n)$ is orthogonal over the square of integration. Therefore, 


$$
\frac{1}{4 \pi^{2}} \int_{0}^{2 \pi} \int_{0}^{2 \pi} S_{n}^{2} d x d y=\left(\frac{1}{8}+\frac{1}{8}+1\right) T_{n}=\frac{5}{4} T_{n}
$$

Since $\left|S_{n}\right| \leq 3 R_{n}$, an application of the second inequality of Lemma (4.2.2) gives

$$
\frac{1}{4 \pi^{2}} \int_{0}^{2 \pi} \int_{0}^{2 \pi} e^{\frac{1}{2} \lambda^{2} S_{n}} d x d y \leq 1+\frac{5}{36} \frac{T_{n}}{R_{n}^{2}} e^{\frac{3}{2} \lambda^{2} R_{n}}
$$

so that, by the hypothesis a) of the theorem, we have finally, a denoting a positive constant,

$$
\int_{0}^{1} I_{n}^{2}(t) d t \leq e^{\frac{1}{2} \lambda^{s} R_{n}}\left(1+\frac{a}{n^{\gamma}} e^{\frac{3}{\lambda^{\lambda} R_{n}}}\right)
$$

Using the inequalities (4.5.2) and (4.5.3), let us apply Lemma (4.2.4) to determine a lower bound for the measure $\left|E_{n}\right|$ of the set $E_{n}$ of points $t$ such that

$$
I_{n}(t) \geq n^{-\eta} e^{\frac{1}{4} \lambda^{2} R_{n}-\lambda^{4} T_{n}},
$$

where the number $\eta>0$ is to be determined later and the factor $n^{-\eta}$ plays role of the $\delta$ of Lemma (4.2.4). The lemma gives immediately

that is to say,

$$
\left|E_{n}\right| \geq\left(1-n^{-\eta}\right)^{2} \frac{e^{\frac{1}{2} \lambda^{n} R_{n}-2 \lambda^{\prime} T_{n}}}{e^{\frac{1}{2 \lambda^{2} R_{n}}}\left(1+\frac{a}{n^{\gamma}} e^{\frac{9}{2} \lambda^{2} R_{n}}\right)},
$$

$$
\left|E_{n}\right| \geq\left(1-2 n^{-\eta}\right) \cdot e^{-2 \lambda^{*} T_{n}} \cdot\left(1-\frac{a}{n^{\gamma}} e^{\frac{8}{2} \lambda^{2} R_{n}}\right) .
$$

Let us now fix a number $\theta, 0<\theta<1$, and let $\lambda=\theta\left(\frac{2}{3} \gamma \frac{\log n}{R_{n}}\right)^{1}$, so that

$$
e^{\frac{8}{2} \lambda^{2} R_{n}-\gamma \log n}=e^{\left(\theta^{2}-1\right) \gamma \log n} \text {. }
$$

One has

$$
2 \lambda^{4} T_{n}=\theta^{4} \frac{8}{9} \gamma^{2} \frac{\log ^{2} n}{R_{n}^{2}} T_{n}<\frac{b \log ^{2} n}{n^{\gamma}}
$$

$b$ denoting a positive constant, so that

$$
e^{-2 \lambda^{\cdot} T_{n}}>1-\frac{b \log ^{2} n}{n^{\gamma}},
$$


and

$$
\left|E_{n}\right| \geq\left(1-\frac{2}{n^{\eta}}\right)\left(1-\frac{b \log ^{2} n}{n^{\gamma}}\right)\left(1-\frac{a}{\left.n^{\gamma / 1}-\theta^{2}\right)}\right)
$$

Choosing now $\eta=\gamma\left(1-\theta^{2}\right)$, we have

$$
\left|E_{n}\right| \geq\left(1-\frac{B}{n^{\gamma\left(1-0^{2}\right)}}\right)
$$

$B$ denoting a constant.

In the set $E_{n}$ one has, by (4.5.4),

so that

$$
e^{\lambda M_{n}(t)}>I_{n}(t)>e^{\frac{1}{1} \lambda^{2} R_{n}-\lambda^{*} T_{n} \cdots \eta \log n},
$$

$$
M_{n}(t)>\frac{\lambda}{4} R_{n}-\lambda^{3} T_{n}-\frac{\eta}{\lambda} \log n=0 \frac{\sqrt{\gamma}}{2 \sqrt{6}}\left(R_{n} \log n\right)^{\downarrow}-\lambda^{3} T_{n}^{\prime}-\frac{\eta}{\lambda} \log n
$$

Now

$$
\begin{aligned}
\lambda^{3} T_{n} & =O\left\{\left(\frac{\log n}{R_{n}}\right)^{\frac{3}{2}} T_{n}^{y}\right\}=O\left\{\log n \cdot \frac{T_{n}}{R_{n}^{2}}\left(R_{n} \log n\right)^{\frac{1}{1}}\right\} \\
& =O\left\{\frac{\log n}{n^{\gamma}}\left(R_{n} \log n\right)^{\frac{1}{t}}\right\}=o\left(R_{n} \log n\right)^{\frac{1}{2}}
\end{aligned}
$$

and

$$
{ }_{\lambda}^{\eta} \log n=\frac{\gamma\left(1-\theta^{2}\right)}{\theta} \sqrt{\frac{3}{2 \gamma}}\left(R_{n} \log n\right)^{1}=6\left(0^{-2}-1\right) \frac{0}{2} \frac{\gamma}{\sqrt{6}}\left(R_{n} \log n\right)^{1}
$$

so that, writing $\varepsilon_{\theta}=6\left(0^{-2}-1\right)$ and fixing $\theta$ close enough to 1 to have $\varepsilon_{\theta}$, say, less than $\frac{1}{2}$, we have for $t \in E_{n}$,

$$
M_{n}(t)>\frac{0 \sqrt{\gamma}}{2 \sqrt{6}}\left(1-\varepsilon_{\theta} \cdot o(1)\right)\left(R_{n} \log n\right)^{\frac{1}{2}}
$$

Let us now take an integer $s$ such that

$$
s \gamma\left(1-\theta^{2}\right)>1
$$

Then the series $\Sigma^{*} n^{-\gamma\left(1-\theta^{2}\right)}$ extended only over the integers $n=m^{s}(m=1,2, \ldots)$ is convergent. Hence, by the lower bound found for $\left|E_{n}\right|$,

$$
\sum_{m=1}^{\infty}\left(1-\left|E_{m} 8\right|\right)<\infty,
$$

and thus, for almost all $t$,

$$
\lim _{n \rightarrow m^{8} \rightarrow \infty} \frac{M_{n}(t)}{\left(R_{n} \log n\right)^{\frac{j}{t}}} \geq \frac{\theta V^{\prime} \gamma}{2 \sqrt{6}}\left(1-\varepsilon_{0}\right)
$$


We must now, in order to complete the proof of the theorem, extend this inequality to the case when $n$ tends to $\infty$ through all integra] values. For this purpose we shall use Lemma (4.4.1) and assumption b) of our theorem.

Denoting by $\mathfrak{M}_{m}^{*}(t)$ the maximum with respect to $n$, for $m^{s}<n \leq(m+1)^{s}$, of

$$
\max _{x}\left|P_{n}(x, t)-P_{m^{s}}(x, t)\right|
$$

we have, by Lemma (4.4.1),

$$
\lim \sup _{m} \frac{\mathfrak{M}_{m}^{\star}(t)}{\left\{\left(R_{(m+1)^{8}}-R_{m^{8}}\right) \log (m+1)^{s}\right\}^{\frac{1}{t}}} \leq 2
$$

so that, since $R_{(m+1)^{s}} / R_{m^{8}} \rightarrow 1$ by assumption b),

$$
\limsup _{m} \frac{\mathfrak{M}_{m}^{\star}(t)}{\left(R_{m^{8}} \log m^{s}\right)^{\frac{1}{2}}}=0
$$

Now, since for $m^{s}<n \leq(m+1)^{s}$ we have

$$
M_{n}(t) \geq M_{m^{8}}(t)-\mathfrak{M}_{m}^{*}(t)
$$

it follows that for almost all $t$,

$$
\begin{aligned}
\lim _{n} \inf \frac{M_{n}(t)}{\left(R_{n} \log n\right)^{t}} & =\lim \inf _{n} \frac{M_{n}(t)}{\left(R_{m^{s}} \log m^{s}\right)^{t}} \\
& \geq \lim \inf \frac{M_{m^{s}}(t)}{\left(R_{m^{s}} \log m^{s}\right)^{t}}-\lim \sup \frac{\mathfrak{M}_{m}^{*}(t)}{\left(R_{m^{s}} \log m^{s}\right)^{t}} \\
& \geq \frac{\theta \sqrt{\gamma} \frac{1}{2 \sqrt{6}}\left(1-\varepsilon_{\theta}\right)}{}
\end{aligned}
$$

It remains now to observe that the last inequality being true for all $\theta, 0<\theta<1$, we can take $\theta$ arbitrarily close to 1 and $\varepsilon_{\theta}$ arbitrarily small, so that

$$
\underset{n}{\lim \inf } \frac{M_{n}(t)}{\left(R_{n} \log n\right)^{t}} \geq \frac{\sqrt{\gamma}}{2 \sqrt{6}}
$$

for almost all $t$, which proves our theorem.

Remarks on Theorem (4.5.1). Let us observe that the preceding argument shows that, with assumption a) alone, one has

$$
\lim _{n} \sup \frac{M_{n}(t)}{\left(R_{n} \log n\right)^{t}} \geq \frac{\sqrt{\gamma}}{2 \sqrt{6}},
$$


so that, comparing this with Theorem (4.2.1) we have at least the true order of magnitude for the superior limit.

We may also add that we do not use in the proof the full force of assumption b), which is needed only when $n_{1}$ and $n_{2}$ are of the form $m^{s}$ and $(m+1)^{s}$.

6. The case $\gamma=1$ deserves special attention. We have then $T_{n} / R_{n}^{2}=O(1 / n)$ and this condition is certainly satisfied if the $r_{m}$ are bounded both above and below (i.e. away from zero). In this case assumption b) of Theorem (4.5.1) is automatically true, if $n_{1}$ and $n_{2}$ are $m^{s}$ and $(m+1)^{s}$ respectively. We shall show that in this case the value of the constant $A$ in Theorem (4.2.1) may be reduced from 2 to 1 .

(4.6.1) Theorem. Let us consider the series (4.1.1) and let $P_{n}, M_{n}, R_{n}, T_{n}$ have the same meaning as before. Then, under the sole assumption $T_{n} / R_{n}^{2}=O(1 / n)$ we have, almost everywhere in $t$,

$$
\frac{1}{2 \sqrt{6}} \leq \lim \inf \frac{M_{n}(t)}{\sqrt{R_{n} \log n}} \leq \lim \sup \frac{M_{n}(t)}{\sqrt{R_{n} \log n}} \leq 1
$$

This is true, in particular, for the series $\Sigma p_{m}(t) \cos m x$.

Proof. From the remark just made it follows that the first inequality (4.6.2) will be proved if we show that, for any integer $s, R_{(m+1)^{8}} / R_{m^{g}} \rightarrow 1$. Now,

$$
\left[R_{(m+1)^{8}}-R_{m^{8}}\right]^{2} \leq\left[(m+1)^{s}-m^{s}\right] T_{(m ; 1)^{8}},
$$

by Schwarz's inequality, so that

as stated.

$$
\left[\begin{array}{c}
R_{(m: 1)^{*}--} R_{m^{*}} \\
R_{(m: 1)^{*}}
\end{array}\right]^{2} \leq \frac{\left[(m+1)^{s}-m^{s}\right] T_{(m ; 1)^{8}}}{R_{(m+1)^{8}}^{2}}=O\left\{\begin{array}{c}
(m+1)^{s}-m^{s} \\
(m+1)^{s}
\end{array}\right\}=o(1)
$$

We now prove the part of the theorem concerning lim sup. We begin as in the proof of Theorem (4.3.1),

but write

$$
\int_{0}^{1} e^{\lambda\left|P_{n}\right|} d t<2 e^{\frac{1}{2} \lambda^{2} \sum_{1}^{n} r_{m}^{2} \cos ^{2} m x}
$$

$$
\sum_{1}^{n} r_{m}^{2} \cos ^{2} m x=\frac{1}{2} R_{n}+\frac{1}{2} \sum_{1}^{n} r_{m}^{2} \cos 2 m x
$$

so that

$$
\int_{0}^{1} d t \int_{0}^{2 \pi} e^{\lambda\left|P_{n}\right|} d x<2 e^{\frac{1}{4} \lambda^{2} R_{n}} \int_{0}^{2 \pi} e^{\frac{1}{4} \lambda^{2} \sum_{1}^{n} r_{m}^{2} \cos 2 m x} d x
$$


Applying now Lemma (4.2.2), we have

$$
\begin{aligned}
\frac{1}{2 \pi} \int_{0}^{2 \pi} e^{\frac{1}{4} \lambda^{2} \sum_{1}^{n} r_{m}^{2} \cos 2 m x} d x & <1+\frac{1}{2} \frac{T_{n}}{R_{n}^{2}} e^{\frac{1}{4} \lambda^{2} R_{n}} \\
& <1+\frac{a}{n} e^{\frac{1}{4} \lambda^{2} R_{n}},
\end{aligned}
$$

$a$ being a positive constant, so that

$$
\int_{0}^{1} d t \int_{0}^{2 \pi} e^{\lambda\left|P_{n}\right|} d x<4 \pi e^{\frac{1}{4} \lambda^{2} R_{n}}\left(1+\frac{a}{n} e^{\frac{1}{4} \lambda^{2} R_{n}}\right) .
$$

Taking $\lambda=2\left(R_{n}^{-1} \log n\right)^{\frac{1}{2}}$ we get

$$
\int_{0}^{1} d t \int_{0}^{2 \pi} e^{\lambda\left|P_{n}\right|} d x<4 \pi(1+a) e^{\log n} .
$$

By Lemma (4.2.3), taking a positive number $\theta$ less than 1 , we get as in the proof of Theorem (4.3.1),

$$
\int_{0}^{1} e^{\theta \lambda M_{n}} d t<\frac{4 \pi n}{1-\theta}(1+a) e^{\log n}=\frac{4 \pi(1+a)}{1-\theta} e^{2 \log n} .
$$

Fixing an $\eta>0$ we have

$$
\int_{0}^{1} e^{\theta \lambda M_{n}-(2: \eta) \log n} d t<\frac{4 \pi(1+a)}{1-\theta} n \eta
$$

Let $s$ be an integer such that $s \eta>1$. Then $\sum m^{-s \eta}<\infty$ so that

$$
\sum_{n=m^{8}}^{\infty} e^{\theta \lambda M_{n} \cdots(2 ; \eta) \log n}<\infty
$$

for almost all $t$, with $\lambda=\lambda_{n}=2\left(R_{n}^{-1} \log n\right)^{l}$. From this we deduce, as in the proof of Theorem (4.3.1), that

$$
\lim _{n=m^{\varepsilon} \rightarrow \infty} \frac{M_{n}(t)}{\left(R_{n} \log n\right)^{\frac{1}{2}}} \leq \theta^{-1}\left(1+\frac{1}{2} \eta\right)
$$

for almost all $t$, and since $\theta$ may be taken arbitrarily close to 1 ,

$$
\lim _{n=m^{8} \rightarrow \infty} \frac{M_{n}(t)}{\left(R_{n} \log n\right)^{\frac{1}{2}}} \leq 1+\frac{1}{2} \eta .
$$


To pass to all values of $n$, we use Lemma (4.4.1), just as in the proof of Theorem (4.5.1). We have, with the same notation, $M_{n} \leq M_{m^{s}}+\mathfrak{M}_{m}^{*}$, for $m^{s}<n \leq(m+1)^{s}$, and it follows immediately that

$$
\lim \sup \frac{M_{n}(t)}{\left(R_{n} \log n\right)^{\frac{1}{2}}} \leq 1+\frac{1}{2} \eta
$$

for almost all $t$. And since $\eta$ is arbitrarily small, our result follows.

Remarks to Theorem (4.6.1). It is not excluded that for series having coefficients of sufficiently regular behavior, in particular for the series $\sum \varphi_{m}(t) \cos m x$, the expression $M_{n} /\left(R_{n} \log n\right)^{\frac{1}{2}}$ (for the particular series, $M_{n} /(n \log n)^{\frac{1}{2}}$ ) tends to a limit, p.p. in $t$, as $n \rightarrow \infty$. We have not, however, been able to prove a result of this kind, or even to narrow the gap between the constants of Theorem (4.6.1).

We shall see that the gap between the constants is reduced if we replace Rademacher's functions by Steinhaus'.

7. We now proceed to generalize the preceding result.

(4.7.1) Theorem. Let $(\alpha, \beta)$ be a fixed interval contained in $(0,2 \pi)$ and let $M_{n}(\alpha, \beta)$ denote the maximum of $\left|P_{n}\right|$ for $\alpha \leq x \leq \beta$. Then, under the same assumptions as in Theorem (4.5.1), and almost everywhere in $t$,

$$
\lim \inf \frac{M_{n}(\alpha, \beta)}{\left(R_{n} \log n\right)^{1}} \geq c(\gamma)
$$

$c(\gamma)$ being a positive constant depending on $\gamma$, which is at least equal to the value $\sqrt{\gamma} / 2 \sqrt{6}$ found for the constant of Theorem (4.5.1).

Proof. The proof follows the pattern of the proof of Theorem (4.5.1), and we only sketch it briefly to indicate the differences. Writing

one has

$$
J_{n}=J_{n}(\alpha, \beta, t)=\frac{1}{\beta-\alpha} \int_{\alpha}^{\beta} e^{\lambda P_{n}} d x
$$

$$
\int_{0}^{1} J_{n} d t \geq e^{\frac{1}{4} \lambda^{2} R_{n}-\lambda^{\prime} T_{n}} \cdot \frac{1}{\beta-\alpha} \int_{\alpha}^{\beta} e^{\frac{1}{4} \lambda^{2} \sum^{n} r_{m}^{2} \cos 2 m x} d x
$$

Now,

$$
\left\{\frac{1}{\beta-\alpha} \int_{\alpha}^{\beta}\left|\sum_{1}^{n} r_{m}^{2} \cos 2 m x\right| d x\right\}^{2} \leq \frac{1}{\beta-\alpha} \int_{\alpha}^{\beta}\left(\sum_{1}^{n} r_{m}^{2} \cos 2 m x\right\}^{2} d x \leq \frac{\pi T_{n}}{\beta-\alpha},
$$


and since

$$
e^{\frac{1}{4} \lambda^{2} \sum_{1}^{n} r_{m}^{2} \cos 2 m x} \geq 1+\frac{1}{2} \lambda^{2} \sum_{1}^{n} r_{m}^{2} \cos 2 m x
$$

one has

$$
\frac{1}{\beta-\alpha} \int_{\alpha}^{\beta} e^{\frac{1}{4} \lambda^{2} \sum_{1}^{n} r_{m}^{2} \cos 2 m x} d x \geq 1-\frac{1}{4} \lambda^{2}\left(\frac{\pi T_{n}}{\beta-\alpha}\right)^{\frac{1}{2}} \geq 1-\frac{\lambda^{2} T_{n}^{\frac{1}{n}}}{(\beta-\alpha)^{\frac{3}{2}}}
$$

so that

$$
\int_{0}^{1} J_{n} d t \geq e^{\frac{1}{4} \lambda^{2} R_{n}-\lambda^{4} T_{n}}\left(1-\frac{\lambda^{2} T_{n}^{\frac{1}{k}}}{(\beta-\alpha)^{\frac{1}{2}}}\right)
$$

We now find an upper bound for $\int_{\alpha}^{\beta} J_{n}^{2} d t$ by using, as in the proof of Theorem (4.5.1), a double integral and find, with the notation of that theorem,

$$
\int_{0}^{1} J_{n}^{2} d t \leq e^{\frac{1}{2} \lambda^{2} R_{n}} \frac{1}{(\beta-\alpha)^{2}} \int_{\alpha}^{\beta} \int_{\alpha}^{\beta} e^{\frac{1}{2} \lambda^{2} S_{n}} d x d y
$$

As in Theorem (4.5.1), we have $\left|S_{n}\right| \leq 3 R_{n}$. Also

$$
\frac{1}{(\beta-\alpha)^{2}} \int_{\alpha}^{\beta} \int_{\alpha}^{\beta} S_{n}^{2} d x d y \leq \frac{1}{(\beta-\alpha)^{2}} \int_{0}^{2 \pi} \int_{0}^{2 \pi} S_{n}^{2} d x d y \leq \frac{4 x^{2}}{(\beta-\alpha)^{2}}{ }_{4}^{5} T_{n}=5 \pi^{2} \cdot \frac{T_{n}}{(\beta-\alpha)^{2}},
$$

so that an application of the first inequality of the Lemma (4.2.2) gives

$$
\begin{aligned}
\frac{1}{(\beta-\alpha)^{2}} \int_{\alpha}^{\beta} \int_{\alpha}^{\beta} e^{\frac{1}{2} \lambda^{2} S_{n}} d x d y & \leq 1+\frac{1}{2} \lambda^{2} \frac{\pi \sqrt{5} \sqrt{T_{n}}}{\beta-\alpha}+\frac{5 \pi^{2} T_{n}}{9(\beta-\alpha)^{2} R_{n}^{2}} e^{\frac{3}{2} \lambda^{2} R_{n}} \\
& \leq 1+\frac{a \lambda^{2} \sqrt{T_{n}}}{\beta-\alpha}+\frac{a T_{n}}{(\beta-\alpha)^{2} R_{n}^{2}} e^{\frac{3}{2} \lambda^{2} R_{n}}
\end{aligned}
$$

$a$ being a positive absolute constant. Hence

$$
\int_{0}^{1} J_{n}^{2} d t \leq e^{\frac{1}{2} \lambda^{2} R_{n}}\left[1+\frac{a \lambda^{2} \sqrt{T_{n}^{-}}}{\beta-\alpha}+\frac{a T_{n}}{(\beta-\alpha)^{2} R_{n}^{2}} e^{\frac{3}{2} \lambda^{2} R_{n}}\right]
$$

From here the proof proceeds as in the case of Theorem (4.5.1), using the inequalities (4.7.2) and (4.7.3) instead of (4.5.2) and (4.5.3), and taking into account 
the hypothesis $T_{n} / R_{n}^{2}=O\left(n^{-\gamma}\right)$. One has only to observe that, since $\lambda=O\left(R_{n}^{-1} \log n\right)^{\frac{1}{2}}$, one has

$$
\lambda^{2} T_{n}^{\mathbf{k}}=O\left\{\frac{\log n}{R_{n}} \cdot T_{n}^{\mathbf{k}}\right\}=O\left(n^{-\mathrm{t} \gamma} \log n\right) .
$$

Once the theorem has been established for a sequence of integers $n=m^{s}$ one proves it for all $n$ by using again Lemma (4.4.1). The constant $c(\gamma)$ can be taken equal to $\sqrt{\gamma} / 2 \sqrt{6}$.

The comments on Theorems (4.5.1) and (4.6.1) are applicable here without change.

By taking the end points $\alpha, \beta$ rational, one sees immediately that if we exclude a certain set of values of $t$ of measure zero then

$$
\liminf _{n} \frac{M_{n}(\alpha, \beta)}{\left(R_{n} \log n\right)^{\frac{3}{2}}} \geq \frac{\sqrt{\gamma}}{2 \sqrt{6}}
$$

for any fixed interval $(\alpha, \beta)$.

It is also easily seen that the theorem holds for the intervals $\left(\alpha_{n}, \beta_{n}\right)$ whose length and position vary with $n$, provided that $\beta_{n}-\alpha_{n}>n^{-\sigma}$, where $\sigma$ is a sufficiently small number which can be determined if $\gamma$ is given. One finds $\sigma<\frac{1}{2} \gamma$, but the constant of the theorem depends then on $\sigma$. The details are left to the reader. Finally, Theorem (4.7.1) holds if the interval $(\alpha, \beta)$ is replaced by a set $E$ of positive measure; it is enough to replace in the proof $\beta-\alpha$ by $|E|$.

8. Some results for the case in which $\sum r_{m}^{2}$ is slowly divergent or is convergent.

If the series $\sum r_{m}^{2}$ diverges slowly, or is convergent, the assumption a) of Theorem (4.5.1) is not satisfied. In order to show what the situation is in that case, we shall consider examples of series with regularly decreasing coefficients.

(i) The series

$$
\sum_{1}^{\infty} m^{-(1-\varepsilon)} \varphi_{m}(t) \cos m x
$$

presents no difficulty since here $T_{n} / R_{n}^{2}$ is $O\left(n^{-4 e}\right)$ if $\varepsilon<1$, is $O\left(n^{-1} \log n\right)$ if $\varepsilon=1$ and is $O\left(n^{-1}\right)$ if $\varepsilon>1$, so that condition a) of Theorem (4.5.1) is satisfied. Since condition b) of that theorem is also satisfied, the exact order of $M_{n}(t)$ is

(ii) The series

$$
\left(R_{n} \log n\right)^{\boldsymbol{t}} \sim n^{e}(\log n)^{\mathbf{t}} .
$$

$$
\sum_{1}^{\infty} m^{-t} \varphi_{m}(t) \cos m x
$$

does not satisfy condition a) of Theorem (4.5.1). By Theorem (4.3.1) we have $M_{n}(t)=O(\log n)$, p.p. in $t$, and we are going to show that this is the exact order. 
We consider the sequence of polynomials

$$
Q_{n}=P_{n}-P_{p}=\sum_{p+1}^{n} m^{-1} \varphi_{m}(t) \cos m x
$$

where $p=p(n)$ is a function of $n$. We take $p=\left[n^{\gamma}\right]$, where $\gamma$ is a positive number less than 1 , to be determined later. We write $M(p, n)=M(p, n, t)=\operatorname{Max}_{x}\left|Q_{n}\right|$, the other notations remaining the same as in Theorem (4.5.1).

We observe that for the polynomials $Q_{n}$ we have

$$
\frac{T_{n}-T_{p}}{\left(R_{n}-R_{p}\right)^{2}}=O\left[\frac{p^{-1}-n^{-1}}{(\log n-\log p)^{2}}\right]=O\left(n^{-\gamma} \log ^{-2} n\right)
$$

so that condition a) of Theorem (4.5.1) holds; it is easily seen that in the proof of the inequality (4.5.5) of Theorem (4.5.1) the fact that the polynomials are partial sums of the form $\sum_{i}^{n}$ is irrelevant, so that the proof of the inequality (4.5.5) applies to the sequence $Q_{n}$ since condition a) is satisfied [condition b) is not required for the proof of that inequality]. We have to replace $T_{n}$ by $T_{n}-T_{p}, R_{n}$ by $R_{n}-R_{p}, M_{n}$ by $M(p, n)$. Hence, for $s \gamma\left(1-\theta^{2}\right)>1$ and for almost all $t$,

$$
\liminf _{n \rightarrow m^{8}} \frac{M(p, n)}{\left\{\left(R_{n}-R_{p}\right) \log n\right\}} \geq \frac{\theta \sqrt{\gamma}}{2 \sqrt{6}}\left(1-\varepsilon_{\theta}\right)
$$

$\theta$ and $\varepsilon_{\theta}$ having the same meaning as in Theorem (4.5.1). Hence

$$
\lim _{n=m^{8}} \frac{M(p, n)}{\log n} \geq \frac{\theta \sqrt{\gamma}}{2 \sqrt{6}}\left(1-\varepsilon_{\theta}\right) \sqrt{1-\gamma}
$$

Now, by Theorem (4.3.1) we have, for almost all $t$,

$$
\lim \sup \frac{M_{p}}{\left(R_{p} \log p\right)^{t}} \leq 2 \text {, i.e. } \lim \sup \frac{M_{p}}{\log p} \leq 2
$$

where, as usual, $M_{p}=\max _{x}\left|P_{p}\right|$. In other words,

so that, if $M_{n}=\max _{x}\left|P_{n}\right|$,

$$
\lim _{p} \sup M_{p} / \log n \leq 2 \gamma
$$

$$
\underset{n=m^{8}}{\lim \inf } \frac{M_{n}}{\log n} \geq \liminf _{n=m^{8}} \frac{M(p, n)}{\log n}-\lim \sup \frac{M_{p}}{\log n} \geq \frac{\theta \sqrt{\gamma}}{2 \sqrt{6}}\left(1-\varepsilon_{\theta}\right) \sqrt{1-\gamma}-2 \gamma
$$

which is a positive quantity if $\gamma$ is small enough. 
We now pass to the sequence of all $n$, like in Theorem (4.5.1), by using Lemma (4.4.1) which can be applied to the proof since plainly for the series $\sum m^{-\frac{1}{2}} \varphi_{n}(t) \cos m x$ one has $R_{n_{1}} / R_{n_{2}} \rightarrow 1$ whenever $n_{1} / n_{2} \rightarrow 1$.

Hence, combining our results and observing that $\theta$ is as close to $l$ as we wish, we have, for almost all $t$

$$
\frac{1}{2 \sqrt{6}} \sqrt{\gamma(1-\gamma)}-2 \gamma \leq \lim \inf M_{n} / \log n \leq \lim \sup M_{n} / \log n \leq 2,
$$

where we can take, e.g., $\gamma=1 / 100$.

The argument could be applied to show that the exact order of magnitude of $M_{n}(t)$ for almost all $t$ is again $\left(R_{n} \log n\right)^{\frac{1}{2}}$ for $r_{m}=m^{-\frac{1}{2}}(\log m)^{\cdots \alpha}$, if $0<\alpha<\frac{1}{2}$, but it breaks down in the case $\alpha=\frac{1}{2}$ which we are now going to consider.

(iii). The series $\sum(m \log m)^{-\frac{1}{2}} \varphi_{m}(t) \cos m x$. In this case the function $\left(R_{n} \log n\right)^{\frac{1}{2}} \sim$ $\sim(\log n \cdot \log \log n)^{\frac{1}{2}}$ does not give the right order of magnitude for $M_{n}$, for we are going to show that in the present case we have $M_{n}=O(V \log n)$ p.p. in $t$.

In fact, setting

$$
\sigma_{n}=\sum_{2}^{n}(m \log m)^{-\frac{1}{2}} \varphi_{m}(t) \cos m x, \quad P_{n}=\sum_{2}^{n} m^{-\frac{1}{2}} \varphi_{m}(t) \cos m x
$$

we have, by Abel's transformation,

$$
\sigma_{n}=\sum_{2}^{n}\left\{\begin{array}{cc}
1 & 1 \\
V \log m & V \log (m+1)
\end{array}\right\} P_{m}+\frac{1}{V \log n} P_{n},
$$

and since $\max _{\tau}\left|P_{m}\right|=O(\log m)$ p.p. in $t$ we have, again p.p. in $t$,

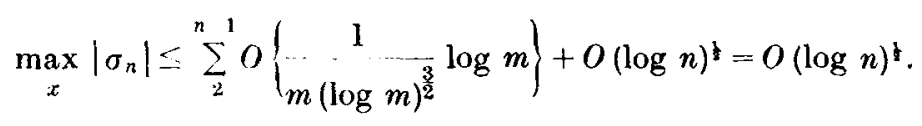

It can also be seen that $M_{n}=\max _{x}\left|\sigma_{n}\right|$ is $o(\log n)^{t}$ for almost no $t$. For suppose that $M_{n}=o(\log n)^{\frac{1}{2}}$ for $t$ belonging to a set $E$ of positive measure. Then

$$
P_{n}=\sum_{2}^{n-1}(\sqrt{\log m}-\sqrt{\log (m+1)}) \sigma_{m}+(\log n)^{\ddagger} \sigma_{n}
$$

and so, in $E$, we would have

$$
\max _{x}\left|P_{n}\right| \leq \sum_{2}^{n \cdots 1}\left\{O\left(\frac{1}{m \sqrt{\log m}}\right) o(\sqrt{\log m})\right\}+o(\log n)=o(\log n),
$$

which we know not to be true.

The same argument can be applied to the more general series

$$
\sum m^{-\frac{t}{2}}(\log m)^{-\alpha} \varphi_{m}(t) \cos m x
$$


if $\frac{1}{2}<\alpha<1$, to show that $M_{n}=O(\log n)^{1-\alpha}$ but not $o(\log n)^{1-\alpha}$, p.p. in $t$ (see also, the end of Chapter $V$, Section 5 ).

\section{Part II. Steinhaus Functions}

9. The problem of the series $\sum_{1}^{\infty} r_{m} e^{i\left(m x+2 \pi \alpha_{m}\right)} \quad\left(r_{m} \geq 0\right)$ is not essentially different from the problem treated in Part $I$, and we shall only indicate the relevant modifications of the argument. They lead to better values for the constants. As mentioned in Section 1, we map the hypercube $0 \leq \alpha_{m} \leq 1(m=1,2, \ldots)$ onto the interval $0 \leq t \leq 1$ using the classical method.

The following result is an analogue of Lemma (4.2.J).

(4.9.1) Lemma. Let $f_{n}=\sum_{i}^{n} c_{m} e^{2 \pi i \alpha_{m}}$, where the $c_{m}$ are complex constants. Then, $\lambda$ being a real number, one has

$$
\int_{0}^{1}\left|e^{\lambda f_{n}}\right| d t=\prod_{1}^{n} J_{0}\left(i \lambda\left|c_{m}\right|\right)
$$

where $J_{0}$ in the Bessel function of order zero:

$$
J_{0}(i z)=\sum_{0}^{\infty} \frac{\left(z^{2} / 4\right)^{k}}{(k !)^{2}}=\int_{0}^{1} e^{z \cos 2 \pi u} d u,
$$

and uriting $C_{n}=\sum_{1}^{n}\left|c_{m}\right|^{2}, D_{m}=\sum_{1}^{n}\left|c_{m}\right|^{4}$, one has

$$
e^{\frac{1}{4} \lambda^{2} C_{n}-\lambda \cdot D_{n}} \int_{0}^{1}\left|e^{\lambda f_{n}}\right| d t \leq e^{\frac{1}{\lambda^{\lambda^{2}} C_{n}}} .
$$

Proot. One has, if $c_{m}=\left|c_{m}\right| e^{2 \pi i \varphi_{m}}$,

$$
\begin{aligned}
\int_{0}^{1}\left|e^{\lambda s_{n}}\right| d t & =\prod_{1}^{n} \int_{0}^{1}\left|e^{\lambda\left|c_{m}\right| e^{2 \pi t\left(\alpha_{m}+\varphi_{m}\right)}}\right| d \alpha_{m} \\
& =\prod_{1}^{n} \int_{0}^{1} e^{\lambda\left|c_{m}\right| \cos 2 \pi\left(\alpha_{m}+\varphi_{m}\right)} d \alpha_{m} \\
& =\prod_{1}^{n} \int_{0}^{1} e^{\lambda\left|c_{m}\right| \cos 2 \pi u} d u=\prod_{1}^{n} J_{0}\left(i \lambda\left|c_{m}\right|\right) .
\end{aligned}
$$


Now, if $z$ is real,

$$
J_{0}(i z) \leq \sum_{0}^{\infty} \frac{1}{k !}\left(\frac{z^{2}}{4}\right)^{k}=e^{\frac{1}{z^{2}}}
$$

and

$$
J_{0}(i z) \geq 1+\frac{1}{4} z^{2} \geq e^{\frac{1}{4} z^{2}-z^{1}} .
$$

Hence

$$
e^{\frac{1}{4} \lambda^{2} C_{n}-\lambda^{2} D_{n}} \leq \prod_{1}^{n} J_{0}\left(i \lambda c_{m}\right) \leq e^{\frac{1}{\lambda^{2} C^{2} C_{n}}},
$$

which proves (4.9.4).

(4.9.5) Lemma. The nolation being the same as in Lemma (4.9.1), one has

$$
\int_{0}^{1} e^{\lambda\left|S_{n}\right|} d t \leq e^{4^{\lambda^{2} C_{n}\left(1+\varepsilon_{n}\right)}}
$$

where $\varepsilon_{n} \rightarrow 0$ if $C_{n} \rightarrow \infty$.

Proof. If $k$ is a positive integer,

$$
\int_{0}^{1}\left|f_{n}\right|^{2 k} d t=\int_{0}^{1} \cdots \int_{0}^{1}\left|f_{n}\right|^{2 k} d \alpha_{1} \ldots d \alpha_{n}=\sum\left(\frac{k !}{k_{1} ! \ldots k_{n} !}\right)^{2}\left|c_{1}\right|^{2 k_{1}} \ldots\left|c_{n}\right|^{2 k_{n}}
$$

the summation being extended over all combinations such that $k_{j} \geq 0, \Sigma k_{j}=k$. Thus

$$
\int_{0}^{1}\left|f_{n}\right|^{2 k} d t \leq\left. k ! \sum_{k_{1} ! \cdots k_{n} !} k ! c_{1}\right|^{2 k_{1}} \cdots\left|c_{n}\right|^{2 k_{n}=k ! C_{n}^{k}}
$$

Hence

$$
\int_{0}^{1} e^{\lambda\left|f_{n}\right|} d t \leq \int_{0}^{1} e^{\lambda\left|f_{n}\right|} d t+\int_{0}^{1} e^{\lambda\left|f_{n}\right|} d t=2 \sum_{0}^{\infty} \frac{\lambda^{2 k}}{(2 k) !} \int_{0}^{1}\left|f_{n}\right|^{2 k} d t \leq 2 \sum_{0}^{\infty}\left(\lambda^{2} C_{n}\right)^{k} \frac{k !}{(2 k) !} .
$$

By Stirling's formula, asymptotically,

$$
\frac{k !}{(2 k) !} \sim \frac{\sqrt{\pi k}}{4^{k} \cdot k !},
$$

so that the general term of the series is

$$
2 \lambda^{2 k} C_{n}^{k} \frac{k !}{(2 k) !} \sim \frac{2\left(\frac{1}{4} \lambda^{2}\right)^{k} C_{n}^{k}}{k !} \sqrt{\pi k},
$$


asymptotically, and it follows that

$$
\int_{0}^{1} e^{\lambda\left|f_{n}\right|} d t \leq e^{\frac{1}{4} \lambda^{2} C_{n}\left(1+\varepsilon_{n}\right)}
$$

where $\varepsilon_{n}$ can be taken as small as we please if $C_{n}$ is large enough.

Let now

$$
P_{n}=\sum_{1}^{n} r_{m} e^{i\left(m x+2 \pi \alpha_{m}\right)}, \quad M_{n}=\underset{x}{\operatorname{Max}}\left|P_{n}\right|, \quad R_{n}=\sum_{1}^{n} r_{m}^{2}, \quad T_{n}=\sum_{1}^{n} r_{m}^{4} .
$$

Theorem (4.3.1) is now replaced by a corresponding theorem for the series $\Sigma r_{m} e^{i\left(m \boldsymbol{x}+2 \pi \alpha_{m}\right)}$, where the constant 2 can be replaced by $\sqrt{2}$, on account of the inequality (4.9.6).

For the proof of the result corresponding to Theorem (4.5.1) we use the integral

$$
H_{n}(t)=\frac{1}{2 \pi} \int_{0}^{2 \pi}\left|e^{\lambda F_{n}}\right| d x
$$

so that Lemma (4.9.1) leads immediately to

$$
\int_{0}^{1} H_{n}(t) d t=\frac{1}{2 \pi} \int_{0}^{2 \pi} d x \int_{0}^{1}\left|e^{\lambda P_{n}}\right| d t \geq e^{\frac{1}{4} \lambda^{\lambda^{2} R_{n} \lambda \cdot T_{n}},}
$$

by inequality (4.9.4).

On the other hand, as in the proof of Theorem (4.5.1),

$$
\int_{0}^{1} H_{n}^{2}(t) d \ell=\frac{1}{4 \pi^{2}} \int_{0}^{2 \pi} \int_{0}^{2 \pi} d x d y \int_{0}^{1}\left|e^{\lambda\left[P_{n}(x, t)+P_{n}(y, t)\right]}\right| d t .
$$

Since

and

$$
P_{n}(x, t)+P_{n}(y, t)=\sum_{1}^{n} r_{m}\left(e^{i m x}+e^{i m y}\right) e^{2 \pi i x_{m}}
$$

$$
\left|e^{i m x}+e^{i m y}\right|^{2}=\left|1+e^{i m(x \cdot y)}\right|^{2}=2(1+\cos m(x-y)),
$$

one has, by (4.9.4),

$$
\int_{0}^{1}\left|e^{\lambda\left[P_{n}(x, t)+P_{n}(y, t)\right]}\right| d t=e^{\frac{1}{2} \lambda^{2} R_{n}+\frac{1}{2} \lambda^{2} \sum_{1}^{n} r_{m}^{2} \cos m(x-y)}
$$

Thus

19-533807. Acta Mathematica. 91. Imprimé le 1 novembre 1954. 


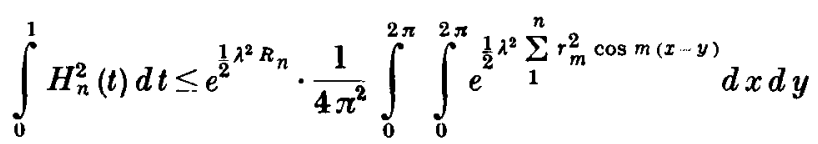

$$
\begin{aligned}
& =e^{\frac{1}{2} \lambda^{2} R_{n}} \cdot \frac{1}{2 \pi} \int_{0}^{2 \pi} e^{\frac{1}{2} \lambda^{2} \sum_{1}^{n} r_{m}^{2} \cos m x} d x
\end{aligned}
$$

so that, by Lemma (4.2.2),

$$
\int_{0}^{1} H_{n}^{2}(t) d t \leq e^{\frac{1}{2} \lambda^{j} R_{n}}\left[1+\frac{T_{n}}{2 R_{n}^{2}} e^{\frac{1}{2} \lambda^{\lambda^{2} R_{n}}}\right] .
$$

The inequalities (4.9.7) and (4.9.8) now lead to the proof of the analogue of Theorem (4.5.1) and, due to the disappearance of the factor 3 in the exponential in the brackets of (4.9.8), the constant $c(\gamma)$ can be taken equal to $\sqrt{\gamma} / 2 \sqrt{2}$.

The analogue of Theorem (4.6.1) is as follows.

(4.9.9) Theorem. Considering the series $\sum_{1}^{\infty} r_{m} e^{i\left(m x+2 \pi \alpha_{m}\right)}$, the expressions $P_{n}, M_{n}$, $R_{n}, T_{n}$ being the same us above, under the sole assumption $T_{n} / R_{n}^{2}=O(1 / n)$, we have, for almost all series,

$$
2^{\stackrel{3}{2}} \leq \lim \inf M_{n} /\left(R_{n} \log n\right)^{\natural} \leq \lim \sup M_{n} /\left(R_{n} \log n\right)^{\natural} \leq 1 .
$$

This applies, in particular, to the series $\sum e^{i\left(m x+2 \pi \alpha_{m}\right)}$.

Remarks. In the proofs of the analogues of Theorems (4.5.1) and (4.6.1) we need, of course, an analogue, for Steinhaus' functions, of Lemma (4.2.5). The proof of the latter, though a little troublesome, follows the same pattern and we sketch it briefly here, the notation being the same as that of Lemma (4.9.1).

Let $k$ be an integer, $n \geq k \geq 1$, and let $(\alpha, \beta)$ be a dyadic interval, $\alpha=p 2^{q}$, $\beta=(p+1) 2^{-a}$, where $q=\frac{1}{2} k(k+1)$. Then it is known (see e.g. Kaczmarz and Steinhaus, Orthogonalreihen, pp. 137-138) that

$$
\int_{\alpha}^{\beta} f_{k}(u) d u=\int_{\alpha}^{\beta} f_{n}(u) d u
$$

and, if both $u$ and $t$ are in the interior of $(\alpha, \beta)$, then

so that

$$
\left|f_{k}(u)-f_{k}(t)\right| \leq 2 \pi\left(\left|c_{1}\right| 2^{-k}+\left|c_{2}\right| 2^{-(k-1)}+\cdots+\left|c_{k}\right| 2^{-1}\right)
$$




$$
\left|\frac{1}{\beta-\alpha} \int_{\alpha}^{\beta} f_{k}(u) d u-f_{k}(t)\right| \leq a C_{k}^{\frac{1}{2}} \leq a C_{n}^{\frac{1}{2}}
$$

and

$$
\begin{gathered}
\left|f_{k}(t)\right| \leq \frac{1}{\beta-\alpha} \int_{\alpha}^{\beta}\left|f_{n}(u)\right| d u+a C_{n}^{\frac{1}{2}} \leq \operatorname{Sup}_{\theta} \frac{1}{\theta-t} \int_{t}^{\theta}\left|f_{n}(u)\right| d u+a C_{n}^{1}, \\
\left|f_{n(t)}(t)\right| \leq f_{n}^{\star}(t)+a C_{n}^{\frac{1}{2}}
\end{gathered}
$$

$f_{n(t)}(t)$ and $f_{n}^{*}(t)$ having the same meaning as in Lemma (4.2.5), and $a$ being an absolute constant.

For $c \geq 2$,

$$
\begin{aligned}
& \left|f_{n(t)}(t)\right|^{q} \leq 2^{q-1}\left\{\left|f_{n}(t)\right|^{q}+a^{q} C_{n}^{\} q}\right\} \\
& \int_{0}^{1}\left|f_{n(t)}(t)\right|^{Q} d t \leq 2^{q-1} \cdot 2\left(\frac{q}{q-1}\right)^{q} \int_{0}^{1}\left|f_{n}(t)\right|^{q} d t+2^{q-1} a^{q} C_{n}^{1^{Q}} \\
& \leq 2^{a+2} \int_{0}^{1}\left|f_{n}(t)\right|^{a} d t+2^{Q-1} a^{a} \int_{0}^{1}\left|f_{n}(t)\right|^{a} d t \leq A^{q} \int_{0}^{1}\left|f_{n}\right|^{a} d t
\end{aligned}
$$

where $A$ is an absolute constant. Hence, by the same sequence of inequalities which led to Lemma (4.2.5),

$$
\int_{0}^{1} e^{\lambda\left|f_{n(t)}(t)\right|} d t \leq 2 e^{t \lambda^{3} A^{2} C_{n}}
$$

The introduction of the constant $A$ in the exponent will lead to the replacement of 2 by $2 A$ in the inequality of Lemma (4.4.1), but clearly will have no effect upon the inequality analogous to (4.5.6).

\section{Chapter V}

\section{Continuity of Trigonometric Series whose Terms have Random Signs}

1. Given a trigonometric series $\sum_{1}^{\infty} r_{m} \varphi_{m}(t) \cos \left(m x-\alpha_{m}\right)$, where $\left\{\varphi_{n}(t)\right\}$ is the Rademacher system and $\Sigma r_{m}^{2}<\infty$, we shall say, briefly, that the series is "randomly continuous" if it represents a continuous function for almost every value of $t$. We propose to give here some new contributions to the theory of such series (which have already been studied). ${ }^{1}$ Without impairing generality we shall simplify writing

I See Paley and Zygmund, loc. cit., and R. SALem, Comptes Rendus, 197 (1933), pp. 113-115 and Essais sur les séries trigonométriques, Paris (Hermann), 1940. 
by considering purely cosine series

$$
\sum_{1}^{\infty} r_{m} \varphi_{n}(t) \cos m x
$$

In what follows, $A$ will denote an absolute constant, not necessarily the same at every occurrence.

(5.1.2) Lemma. Let $Q=Q(t, x)$ denote the polynomial $\sum_{p+1}^{n} r_{m} \varphi_{m}(t) \cos m x$, and let $M=M(t)=\max _{x}|Q(t, x)|$. Let $R=\sum_{p+1}^{n} r_{m}^{2}$. Then $\int_{0}^{1} M d t \leq A(R \log n)^{\frac{1}{t}}$.

Proof. By the argument of Theorem (4.3.1),

Hence

$$
\int_{0}^{1} e^{\theta \lambda M} d t \leq \frac{4 \pi}{1-\theta} e^{\frac{1}{2} \lambda^{2} R_{n} \log n} \quad(0<\theta<1)
$$

$$
e^{\theta \lambda \int_{0}^{1} M d t} \leq e^{\frac{1}{2} \lambda^{2} R+\log n+\log \frac{4 \pi}{1-\theta}}
$$

Taking $\lambda=\left(2 R^{1} \log n\right)^{t}$, we get

$$
\begin{aligned}
\int_{0}^{1} M d t & \leq \theta^{\cdot 1}(2 R \log n)^{t}+\theta^{-1} \log \left(\frac{4 \pi}{1-\theta}\right) \cdot\left(\frac{R}{2 \log n}\right)^{t} \\
& =\theta^{-1}(2 R \log n)^{t}\left[1+\log \left(\frac{4 \pi}{1-\theta}\right) \cdot \frac{1}{2 \log n}\right] \\
& \leq A(R \log n)^{t}
\end{aligned}
$$

which proves the theorem. We see that we could take $A$ as close to $\sqrt{2}$ as we wish if $n$ is large enough, but this is irrelevant for our purposes.

We could also, by writing

$$
\cos m x=\cos p x \cos (m-p) x-\sin p x \sin (m-p) x
$$

prove easily that

$$
\int_{0}^{1} M d t \leq A\{R \log (n-p)\}
$$

but we shall not make use of this slightly stronger inequality. 
(5.1.3) Lemma. Let

$$
Q_{n}=\sum_{p+1}^{n} r_{m} \varphi_{m}(t) \cos m x, \quad M_{n}=\max _{x}\left|Q_{n}\right|
$$

and let, for fixed $m$,

$$
M^{*}(t)=\max _{n} M_{n}(t) \quad \text { when } \quad p+1<n \leq m .
$$

Then, with $R=\sum_{p \rightarrow 1}^{m} r_{k}^{2}$, we have

$$
\int_{0}^{1} M^{*} d t \leq A(R \log m)^{\frac{t}{2}}
$$

Proof. By the argument of Lemma (4.4.1) we have

$$
\int_{0}^{1} e^{\theta \lambda M^{*}} d t \leq \frac{32 \pi}{1-\theta} e^{\frac{1}{2} \lambda^{2} R+\log n}, \quad 0<\theta<1,
$$

which leads, exactly as in the proof of the preceding lemma, to (5.1.4).

(5.1.5) Theorem. Let $R_{n}$ denote 1 the remainder $\sum_{n+1}^{\infty} r_{m}^{2}$ of the convergent series $\sum r_{m}^{2}$. If $\sum n^{1}(\log n)^{-1} \sqrt{ } \bar{R}_{n}^{-}<\infty$, the series (5.1.1) is randomly continuous, Moreover, for almost every $t$ that series converges uniformly in $x$.

Proof. Let us divide the series into blocks $Q_{0}, Q_{1}, \ldots$ such that

$$
Q_{k}=\sum_{n_{k}+1}^{n_{k+1}} r_{m} \varphi_{m}(l) \cos m x \quad\left(n_{0}=0, n_{k}=2^{2^{k}}\right)
$$

Let $M_{k}=\max _{x}\left|Q_{k}\right|$. By lemma (5.1.2),

$$
\int_{0}^{1} M_{k} d t \leq A\left(2^{k} R_{2^{2}}\right)^{t}
$$

By Cauchy's theorem, the convergence of $\Sigma n^{-1}(\log n)^{-} \sqrt{\boldsymbol{R}_{n}}$ implies that of $\Sigma k^{-} \sqrt{R_{2} k}$ which, in turn, implies the convergence of $\Sigma\left(2^{k} R_{2^{2}}\right)^{t}$. Hence $\Sigma \int_{0}^{1} M_{k} d t<\infty$, which shows that $\Sigma M_{k}$ converges for almost every $t$, i.e. for almost every $t$ the series $\Sigma Q_{k}$ converges uniformly in $x$ and the first part of the theorem has been established.

1 Attention of the reader is called to the fact that $R_{n}$, and later on $T_{n}$, has not the same meaning here as in the preceding chapter. 
To prove the second part of the theorem it is enough to observe that, writing

$$
M_{k}^{*}(t)=\max _{n_{k}+1<m \leq n_{k+1}}\left\{\max _{x}\left|\sum_{n_{k}+1}^{m} r_{n} \varphi_{n}(t) \cos n x\right|\right\}
$$

one has, by Lemma (5.1.3),

$$
\int_{0}^{1} M_{k}^{*} d t \leq A\left(2^{k} R_{2^{2}}\right)^{\frac{1}{2}},
$$

so that, for almost all $t, \Sigma M_{k}^{*}<\infty$, and so also $M_{k}^{*} \rightarrow 0$.

2. Remarks on Theorem (5.1.5). a) The condition $\sum n^{-1}(\log n)^{-1} \sqrt{R_{n}}<\infty$ 1s merely sufficient, but not necessary, for the random continuity of the series (5.1.1). It is enough to consider the series $\Sigma p^{-2} \varphi_{2^{2}}{ }^{p}(t) \cos 2^{2^{p}} x$.

b) On the other hand, the condition $\Sigma n^{-1}(\log n)^{-} \sqrt{R_{n}}<\infty$ is the best possible of its kind. In other words, there exist series (5.1.1) which represent a continuous function for no value of $t$ and such that

$$
\Sigma \frac{\sqrt{R_{n}}}{n(\log n)^{\dagger} \omega(n)}<\infty,
$$

$\omega(n)$ being a given function, increasing to $\infty$ with $n$, as slowly as we please.

To see this, let us consider the series $\Sigma \frac{1}{p \psi(p)} \varphi_{2} p(t) \cos 2^{p} x$, where $\psi(p)$ increases to $\infty$ with $p$ but $\Sigma 1 / p \psi(p)=\infty$. The series being lacunary, it cannot represent a continuous function for any value of $t$. But

so that

$$
R_{2} p=\sum_{p+1}^{\infty} \frac{1}{k^{2} \psi^{2}(k)}<\frac{1}{p \psi^{2}(p)}
$$

$$
\left(\frac{R_{2} k}{k}\right)^{t} \cdot \frac{1}{\Omega(k)}<\frac{1}{k \psi(k) \Omega(k)}
$$

Now, no matter how slowly $\Omega(k)$ increases, we can find $\psi(k)$ such that

$$
\Sigma 1 / k \psi(k)=\infty, \quad \Sigma 1 / k \psi(k) \Omega(k)<\infty .
$$

and this proves the statement, if we set $\omega\left(2^{k}\right)=\Omega(k)$ and apply Cauchy's theorem.

c) A necessary condition for random continuity of the series (5.1.1) is known (see Paley and Zygmund, loc. cit.). Let us divide the series into blocks 


$$
P_{k}=\sum_{2^{k_{+}}}^{2^{k+1}} r_{m} \varphi_{m}(t) \cos m x
$$

and let

$$
\Delta_{k}=\sum_{2^{k}+1}^{2^{k+1}} r_{m}^{2}
$$

The condition $\Sigma \sqrt{\Delta_{k}}<\infty$ is necessary, for random continuity.

But the condition is not sufficient, as seen on the following example.

It will be slightly simpler to deal with a series of exponentials $\Sigma r_{m} \varphi_{m}(t) e^{i m x}$. We shall construct a series of this type which for no value of $t$ does represent a continuous function, although the series $\Sigma \Delta_{k}^{\frac{1}{k}}$ will be convergent.

We shall make use of the familiar fact, namely, that if $\psi_{1}, \psi_{2}, \ldots, \psi_{N}$ are any distinct Rademacher functions, then

$$
\max _{x}\left|\sum_{1}^{N} \psi_{k}(t) e^{i 2^{k} x}\right| \geq A N
$$

no matter what value we give to $t$. Let us now determine the coefficients $r_{m}$ in each polynomial $P_{k}$ as follows (compare (5.2.1) and (5.2.2)):

$$
\begin{array}{ll}
r_{m}=k^{-1} & \text { for } m=2^{k}+2^{s} \quad(s=1,2, \ldots, k) \\
r_{m}=0 & \text { for other values of } m .
\end{array}
$$

Then, by the remark just made, we have for all $t$,

while

$$
\max _{x}\left|P_{k}\right|>A k \cdot k^{-1}=A k^{3}
$$

$$
\Delta_{k}=k \cdot k^{-\frac{3}{2}}=k^{-1},
$$

so that $\Delta_{k}^{k}=k^{-1}$. Let us now consider an increasing sequence of integers $n_{q}$ such that $\sum n_{q}^{-t}<\infty$ (e.g. $n_{q}=q^{5}$ ) and construct the series

$$
P_{n_{1}}+P_{n_{\mathbf{z}}}+\cdots+P_{n_{q}}+\cdots
$$

with the polynomials just defined. The series, having infinitely many Hadamard gaps, must, if it represents a bounded function, have its partial sums of order corresponding to the beginning or end of the gaps uniformly bounded. ${ }^{1}$ In particular, $P_{n_{q}}$ must be uniformly bounded. Since $\max \left|P_{n_{q}}\right|>A n_{q}^{\ddagger}$, this is impossible, no matter what value we give to $t$. And yet, for this series, $\Sigma \Delta_{k}^{t}=\Sigma n_{q}^{-t}<\infty$. 
We shall return to the problem of necessary and sufficient conditions for random continuity at the end of this chapter.

3. (5.3.1) Theorem. Suppose that the series (5.1.1.) is randomly continuous. Denote by $\left\{n_{q}\right\}$ any lacunary sequence of positive integers (i.e. such that $n_{a+1} / n_{q} \geq \lambda>1$ ). Write $S_{n}=\sum_{1}^{n} r_{m} \varphi_{m}(t) \cos m x$. Then, for almost every $t$, the partial sums $S_{n_{q}}$, of order $n_{a}$, converge uniformly in $x$.

Proof. Observe first that, $t_{0}$ being a fixed number-not a dyadic rational-the series

$$
\sum r_{m} \varphi_{m}\left(t_{0}\right) \varphi_{m}(t) \cos m x
$$

is randomly continuous, if (5.1.1) is. For let $E$ be the set of measure 1 such that, when $t \in E,(5.1 .1)$ represents a continuous function. Let $t \in E$ and define $t^{\prime}$ by

$$
\varphi_{m}\left(t^{\prime}\right) \varphi_{m}\left(t_{0}\right)=\varphi_{m}(t)
$$

It is easy to see (e.g. by the consideration of dyadic intervals) that the set of $t^{\prime}$ corresponding to the $t \in E$ is also of measure 1 .

Let us now divide the series (5.1.1) into blocks $P_{q}=\sum_{n_{q}+1}^{n_{q}} r_{m} \varphi_{m}(t) \cos m x$ and consider the two series

$$
\begin{aligned}
& P_{0}+P_{1}+P_{2}+P_{3}+\cdots \\
& P_{0}-P_{1}+P_{2}-P_{3}+\cdots
\end{aligned}
$$

It follows from our remarks that the series $P_{0}+P_{2}+P_{4}+\cdots$ and $P_{1}+P_{3}+P_{5}+\cdots$ are both randomly continuous. But both series are series with Hadamards gaps, so that the partial sums $S_{n_{q}}$ of order $n_{q}$ of the series (5.1.1) converge uniformly in $x$, for almost every $t$.

Remark on Theorem (5.3.1). Iset us consider alongside (5.1.1) the series (5.3.2), where now $\varphi_{m}\left(t_{0}\right)=1$ for $m=n_{q}(q=1,2, \ldots)$ and $\varphi_{m}\left(t_{0}\right)=--1$ for $m \neq n_{q}\left(n_{a+1} / n_{q} \geq \lambda>1\right)$. An application of the preceding argument leads to the conlusion that the random continuity of (5.1.1) implies the random continuity of $\Sigma r_{n_{q}} \varphi_{n_{q}}(t) \cos n_{q} x$. The sequence $\left\{n_{q}\right\}$ being lacunary this implies that $\sum\left|r_{n_{q}}\right|<\infty$. In other words, if (5.1.1) is randomly continuous, the moduli of any lacunary subsequence of its coefficients have a finite sum. This is of course a consequence of the necessary condition discussed in Section 2, c), but the proof given here is much simpler. 
4. (5.4.1) Thcorem. Suppose that the series (5.1.1) is randomly continuous. Then, writing

$$
R_{n}=\sum_{n+1}^{\infty} r_{m}^{2}, \quad T_{n}=\sum_{n+1}^{\infty} r_{m}^{4}
$$

we necessarily have $R_{n} \log T_{n} \rightarrow 0$ as $n \rightarrow \infty$

Proof. Let us observe first that the series (5.1.1) converges almost everywhere in $x$ for almost every $t$ (see e.g. Zygmund, Trigonometrical Series, p. 125). Let $Q_{n}=\sum_{n+1}^{\infty} r_{m} \varphi_{m}(t) \cos m x$. By lemma (4.2.1),

$$
\int_{0}^{1} \int_{0}^{2 \pi} e^{\lambda \sum_{n+1}^{N} r_{m} \varphi_{m}(t) \cos m x} d t d x \leq 2 \pi e^{\frac{1}{2} x^{2 ! R} R_{n}}
$$

so that, by the theorems of Fatou and Fubini, $(2 \pi)^{1} \int_{0}^{2 \pi} e^{2 \cdot \theta_{n}} d x=I_{n}(t)$ exists for almost all $t$ and is integrable.

The argument used in the proof of Theorem (4.5.1) can then be applied without change, though $Q_{n}$ is not a polynomial here but an infinite series (see footnote to Lemma (4.2.1)), and we get

$$
\begin{aligned}
& \int_{0}^{1} I_{n} d t \geq e^{1^{\lambda^{2} R_{n}-\lambda^{2} T_{n}},} \\
& \int_{0}^{1} I_{n}^{2} d t \leq e^{\frac{1}{2} \lambda^{2} R_{n}}\left[1+A \frac{T_{n}}{R_{n}^{2}} e^{\frac{3}{2} \lambda^{2} R_{n}}\right] .
\end{aligned}
$$

Taking $\lambda=\left\{\frac{2}{3} R_{n}^{-1} \log \left(R_{n}^{2} / T_{n}\right)\right\}^{1}$, we have

$$
\begin{aligned}
& \int_{0}^{1} I_{n}(t) d t \geq e^{\frac{1}{4} \lambda^{2} R_{n}-\lambda^{\prime} T_{n}} . \\
& \int_{0}^{1} I_{n}^{2}(t) d t \leq(1+A) e^{\frac{1}{2} \lambda^{2} R_{n}},
\end{aligned}
$$

so that, by Lemma (4.2.4), if we denote by $E_{n}$ the set of points $t$ such that

we have

$$
I_{n} \geq \frac{1}{2} e^{\frac{1}{4} \lambda^{2} R_{n} \cdots \lambda \cdot T_{n}}
$$

$$
\left|E_{n}\right| \geq[4(A+1)]^{-1} e^{-2 \lambda \cdot T_{n}} \text {. }
$$


Now,

$$
2 \lambda^{4} T_{n}=\frac{8}{9} R_{n}^{-2} T_{n} \log ^{2}\left(R_{n}^{2} / T_{n}\right) \leq u^{-1} \log ^{2} u,
$$

where $u=R_{n}^{2} / T_{n} \geq 1$, so that $2 \lambda^{4} T_{n} \leq B, B$ being an absolute constant, and

$$
\left|E_{n}\right| \geq\left[4(A+1) e^{B}\right]^{-1}=\delta,
$$

$\delta$ being a positive absolute constant.

Now, writing $M_{n}=M_{n}(t)=\max \left|Q_{n}(t, x)\right|$ for a given $t$ for which $I_{n}(t)$ exists $\left(M_{n}\right.$ can be $\left.+\infty\right)$ one has, if $t \in E_{n}$,

that is to say,

$$
e^{\lambda M_{n}} \geq I_{n}(t) \geq e^{\frac{1}{4} \lambda^{2} R_{n} \cdots \lambda^{\prime} T_{n}-\log 2},
$$

$$
M_{n} \geq \frac{\lambda}{4} R_{n}-\lambda^{3} T_{n}-\frac{\log 2}{\lambda} .
$$

Now,

$$
\begin{gathered}
\frac{\lambda}{4} R_{n}=\frac{1}{2 \sqrt{6}}\left\{R_{n} \log \left(R_{n}^{2} / T_{n}\right)\right\}^{\mid} \\
\lambda^{3} T_{n}<T_{n} R_{n}^{-\frac{3}{2}} \log ^{\frac{3}{2}}\left(R_{n}^{2} / T_{n}\right)=\frac{\log \left(R_{n}^{2} / T_{n}\right)}{R_{n}^{2} / T_{n}}\left\{R_{n} \log \left(R_{n}^{2} / T_{n}\right)\right\}^{*}
\end{gathered}
$$

and

(5.4.4) $\quad \lambda^{1} \log 2=\log 2 \cdot\left(\frac{3}{2}\right)^{\dagger} R_{n}^{\dagger} \log ^{-1}\left(R_{n}^{2} / T_{n}\right)=\left(\frac{3}{2}\right)^{\dagger} \cdot \log 2 \cdot \frac{\left\{R_{n} \log \left(R_{n}^{2} / T_{n}\right)\right\}^{\dagger}}{\log \left(R_{n}^{2} / T_{n}\right)}$.

Suppose now that $R_{n} \log R_{n}^{2} / T_{n}$ does not tend to zero. Since $R_{n} \rightarrow 0$, we see that $R^{2} / T_{n}$ is unbounded. Hence we can find a sequence $\left\{n_{q}\right\}$ of integers with the following properties:
a) $R_{n_{q}}^{2} / T_{n_{q}}$ increases to $+\infty$
b) $R_{n_{q}}\left(\log R_{n_{q}}^{2} / T_{n_{q}}\right) \geq c>0$
c) $n_{q+1} / n_{q} \geq 2$ for all $q$.

It follows then from (5.4.2), (5.4.3), (5.4.4) that $M_{n_{q}}(t)>\sqrt{c} / 10$ in a set $E_{n_{q}}$ of measure $\geq \delta$. But this is impossible if $(5.1 .1)$ is randomly continuous. In fact, $\left\{n_{a}\right\}$ being lacunary, $Q_{n_{q}}$ must tend then to zero for almost every $t$ uniformly in $x$, by the preceding theorem. Now, consider the set $\mathcal{E}$ of points $t$ for which $Q_{n_{q}} \rightarrow 0$ uniformly in $x$. Every $t \in \mathcal{E}$ must belong to all the complementary sets $C E_{n_{q}}$ after a certain rank. Hence

$$
\mathcal{E}=\prod_{1}^{\infty} C E_{n_{q}}+\prod_{2}^{\infty} C E_{n_{q}}+\cdots
$$

If we denote the products on the right by $F_{1}, F_{2}, \cdots$, respectively, then

$$
F_{1} \subset F_{2} \subset \cdots \subset F_{k} \subset \cdots,\left|F_{k}\right| \leq\left|C E_{n_{k}}\right| \leq 1-\delta .
$$


Thus $|\mathcal{E}| \leq 1-\delta$, so that, since obviously $\mathcal{E}$ must be of measure 0 or 1 , we have $|\mathcal{E}|=0$. In other words, if $R_{n} \log R_{n}^{2} / T_{n}$ is not $o(1)$, almost no series (5.1.1) represents a continuous function.

The proof of the theorem is completed by observing that $R_{n} \rightarrow 0$ implies $R_{n} \log R_{n} \rightarrow 0$ so that the condition $R_{n} \log \left(R_{n}^{2} / T_{n}\right) \rightarrow 0$ is equivalent to $R_{n} \log T_{n} \rightarrow 0$.

Corollary. If $\left\{r_{m}\right\}$ is a decreasing sequence, the condition $\mathrm{E} r_{m}^{2}<\infty$ implies $m r_{m}^{2} \rightarrow 0$ so that, for $n$ large enough,

$$
T_{n}<(n+1)^{-2}+(n+2)^{2}+\cdots<1 / n,
$$

and so $R_{n} \log n \rightarrow 0$ is a necessary condition for random continuity. ${ }^{1}$ This is of course true, more generally, if $\sum_{n+1}^{\infty} r_{m}^{4}=O\left(n^{-\varepsilon}\right)$ for some $\varepsilon>0$.

5. We shall now indicate a case of "regularity" in which the convergence of $\sum n^{-1}(\log n)^{-1} \sqrt{R_{n}}$ is both necessary and sufficient for the random continuity of (5.11.1).

(5.5.1) Theorem. If the sequence $\left\{r_{m}\right\}$ is decreasing and if there exists a $p>1$ such that $R_{n}(\log n)^{p}$ is increasing, then the convergence of $\Sigma n^{1}(\log n)^{-1} \sqrt{R_{n}}$ is both necessary and sufficient for the random continuity of (5.1.1).

In view of Theorem (5.1.5) it is sufficient to prove the necessity of the condition.

The hypothesis is better understood if we observe that the boundedness of $\boldsymbol{R}_{n}(\log n)^{p}$ for some $p>1$ implies $\sum n^{-1}(\log n)^{-1} V \boldsymbol{R}_{n}<\infty$. Thus we have to assume that $R_{n}(\log n)^{p}$ is unbounded; our "regularity" condition consists in assuming the monotonicity of the latter expression for some $p>1$.

(5.5.2) Lemma. If (5.5.1) is randomly continuous and if two following conditions are satisfied

$$
\frac{R_{\mathbf{2}^{k}-R_{\mathbf{2}^{k ! 1}}}}{\boldsymbol{R}_{\mathbf{2}^{k}}}=O\left(\frac{1}{k}\right), \quad k R_{\mathbf{2}^{k}}=O(1),
$$

then $\sum n^{-1}(\log n)^{-1} \sqrt{R_{n}}<\infty$.

Proof of the Lemma. Using the notation of Section 3,

$$
\Delta_{k}=R_{\mathbf{2}^{k}}-R_{\mathbf{2}^{k+1}}
$$

we know (by the result of Paley and Zygmund quoted there) that if (5.1.1) is randomly continuous, then $\Sigma \Delta_{n}^{t}<\infty$. Now

1 In particular, the series, $\sum m^{-\frac{1}{2}}(\log m)^{1} \varphi_{m}(t) \cos m x$, for which $R_{n} \log n$ is bounded but does not tend to zero, is not randomly continuous. 


$$
\begin{aligned}
\sum_{1}^{n} k^{-\frac{1}{2}} R_{\mathbf{2}^{k}} & =\sum_{1}^{n-1} O\left(k^{\frac{1}{2}}\right)\left[\sqrt{\boldsymbol{R}_{\mathbf{2}^{k}}}-\sqrt{\boldsymbol{R}_{\mathbf{2}^{k+1}}}\right]+O\left(n \boldsymbol{R}_{\mathbf{2}^{n}}\right)^{\frac{1}{2}} \\
& =\sum_{1}^{n-1} O\left(k^{\frac{1}{2}} \frac{\Delta_{k}}{\sqrt{\boldsymbol{R}_{\mathbf{2}^{k}}}}\right)+O\left(n R_{\left.\mathbf{2}^{n}\right)^{\frac{1}{2}}}\right. \\
& =\sum_{1}^{n-1} O\left(\Delta_{k}^{\frac{1}{2}}\right)+O(1)
\end{aligned}
$$

so that $\Sigma k^{-\frac{1}{2}} \sqrt{R_{2^{k}}}<\infty$ and the lemma follows by an application of Cauchy's theorem.

Proof of Theorem (5.5.1). The sequence $\left\{r_{m}\right\}$ being decreasing, the random continuity of (5.1.1) implies $R_{n} \log n \rightarrow 0$ (see the Corollary of Theorem (5.4.1)). In particular, $k R_{2^{k}} \rightarrow 0$.

Moreover, since $R_{n}(\log n)^{p}$ increases,

$$
\begin{aligned}
& R_{2^{k}} k^{p}<R_{2^{k+1}}(k+1)^{p} \\
& R_{2^{k+1}} / R_{2^{k}}>\left(\frac{k}{k+1}\right)^{p}>1-\frac{A}{k} .
\end{aligned}
$$

Hence $1-\left(R_{2^{k: 1}} / R_{2^{k}}\right) \leq A / k$, and the theorem follows from the lemma.

6. It is clear that the results of this chapter hold when the Rademacher functions are replaced by those of Steinhaus, viz. for the series of the type $\sum_{1}^{\infty} r_{m} e^{i\left(m x+2 \pi \alpha_{m}\right)}$. In particular if $r_{m}>0$ is decreasing, $R_{m} \log m=o(1)$ is necessary for random continuity. It might be interesting to recall in this connection that, if the sequence $\left\{1 / r_{m}\right\}$ is monotone and concave, no matter hon slow is the convergence of $\Sigma r_{m}^{2}$, there always exists a particular sequence $\left\{\alpha_{m}\right\}$ such that the series $\sum_{1}^{\infty} r_{m} e^{i\left(m x+2 \pi \alpha_{m}\right)}$ converges uniformly (see Salem, Comptes Rendus, 201 (1935), p. 470, and Essais sur les séries trigonométriques, Paris (Hermann), 1940), although the series need not be randomly continuous, e.g. if $R_{n} \log n \neq o(1)$.

The problem whether an analogous result holds for the series of the type $\sum_{1}^{\infty} r_{m} \cos m x \varphi_{m}(t)$, where $\left\{\varphi_{m}\right\}$ is the sequence of Rademacher functions, is open.

\section{CH A P T E R V I}

\section{The Case of Power Polynomials}

1. Let us consider a power series $\sum_{0}^{\infty} \alpha_{k} x^{k}$ of radius of convergence 1 and let us also consider the power series $\sum^{\infty} \alpha_{k} \varphi_{k}(t) x^{k}$ and its partial sums 


$$
P_{n}=\sum_{0}^{n} \alpha_{k} \varphi_{k}(t) x^{k}
$$

where $\varphi_{0}, \varphi_{1}, \varphi_{2}, \ldots$ is the sequence of Rademacher functions. We may consider the problem of the order of magnitude, for almost all $t$, of

$$
M_{n}(t)=\max _{-1 \leq x \leq+1}\left|P_{n}\right|
$$

assuming, for the sake of simplicity, that the coefficients $\alpha_{k}$ are real.

From Theorem (4.3.1), using the principle of maximum, we see at once that

$$
M_{n}(t)=O\left(R_{n} \log n\right)^{t}
$$

almost everywhere in $t$, with $R_{n}=\sum_{0}^{n} \alpha_{k}^{2}$. We shall see, however, that better estimates than that can be found and that the problem has some curious features distinguishing it from the corresponding problem for trigonometric polynomials.

(6.1.1) Theorem. If $R_{n} \rightarrow \infty$ and

then

$$
\alpha_{k}^{2}=o\left\{R_{k} / \log \log R_{k}\right\}
$$

$$
\lim \sup M_{n}(t) /\left\{2 R_{n} \log \log R_{n}\right\}^{t}=1
$$

for almost every $t$. On the other hand,

$$
\lim \inf M_{n}(t) / R_{n}^{\ddagger}=O(1),
$$

almost everywhere in $t$.

Thus, unlike in the theorems of Chapter IV, even in the simplest cases (e.g. for $\alpha_{0}=\alpha_{1}=\cdots=1$ ) the maximum $M_{n}(t)$ has no definite order of magnitude p.p. in $t$.

Proof. The inequality $(6.1 .2)$ is a rather simple consequence of the Law of the Iterated Logarithm.

For let $M_{n}^{\prime}(t)$ and $M_{n}^{\prime \prime}(t)$ denote the maximum of $\left|P_{n}\right|$ on the intervals $0 \leq x \leq 1$ and $-1 \leq x \leq 0$ respectively. It is enough to prove (6.1.2) with $M_{n}(t)$ replaced by $M_{n}^{\prime}(t)$. For then the inequality will follow for $M_{n}^{\prime \prime}(t)$ (since it reduces to the preceding case if we replace $\alpha_{k}$ by $\left.(-1)^{k} \alpha_{k}\right)$, and so also for $M_{n}(t)=\max \left\{M_{n}^{\prime}(t), M_{n}^{\prime \prime}(t)\right\}$.

Let us set

Since

$$
S_{m}(t)=\sum_{0}^{m} \alpha_{k} \varphi_{k}(t), \quad S_{n}^{*}(t)=\max _{1 \leq m \leq n}\left|S_{m}(t)\right| .
$$

$$
P_{n}=\sum_{0}^{n} \alpha_{k} \varphi_{k}(t) x^{k}=\sum_{0}^{n-1} S_{k}\left(x^{k}-x^{k+1}\right)+x^{n} S_{n}
$$


we immediately obtain

$$
M_{n}(t) \leq S_{n}^{*}(t)
$$

On the other hand,

$$
S_{n}^{*}(t)=\left|S_{m}(t)\right| \quad \text { for some } m=m(n) \leq n,
$$

so that

$$
\lim _{n} \sup M_{n}(t) /\left(2 R_{n} \log \log R_{n}\right)^{\frac{1}{2}} \leq \lim _{m} \sup \left|S_{m}(t)\right| /\left(2 R_{n} \log \log R_{n}\right)^{\frac{1}{2}} \leq \mathrm{l}
$$

by the Law of the Iterated Logarithm, and this gives (6.1.2). with ' $=$ ' replaced by ' $\leq$ '.

The opposite inequality follows from the fact that $M_{n}(t) \geq\left|S_{n}(t)\right|$ and that $\lim \sup \left|S_{n}(t)\right| /\left(2 R_{n} \log \log R_{n}\right)^{\frac{1}{2}}=1$ p.p. in $t$.

As regards (6.1.3), it is enough to prove it with $M_{n}$ replaced by $S_{n}^{*}$, on account of (6.1.4). By Lemma (4.2.5),

$$
\int_{0}^{1} e^{\lambda s_{n}^{*}} d t \leq 16 e^{\frac{1}{2} \lambda^{2} R_{n}}
$$

Let us consider any function $\omega(n)$ increasing to $+\infty$ with $n$. In the inequality

$$
I_{n}=\int_{0}^{1} e^{\lambda S_{n}^{*} \omega(n)} d t \leq 16 e^{\lambda^{2} R_{n}-\omega(n)}
$$

we set $\lambda-R_{n}^{-\frac{1}{2}} \omega^{\frac{1}{2}}(n)$. Then $I_{n} \leq \exp \left\{-\frac{1}{2} \omega(n)\right\}$. Thus, if $\left\{n_{j}\right\}$ increases fast enough, we have $\sum I_{n_{j}}<\infty$ so that, for almost all $t$ and for $n=n_{j}$ large enough, we shall have $\lambda s_{n}^{*} \leq \omega(n)$, that is

$$
\lim \inf _{n} S_{n}^{*}(t) /\left\{R_{n} \omega(n)\right\}^{t} \leq 1, \quad \text { p.p. in } t .
$$

From this it is easy to deduce the validity of (6.1.3), with $M_{n}$ replaced by $S_{n}^{*}$, for almost every $t$. For suppose that (6.1.3) does not hold in a set $E$ of positive measure. Then $S_{n}^{*}(t) / R_{n}^{\ddagger}$ tends to infinity in $E$. Using the theorem of Egoroff, we may assume that this convergence to $\infty$ is uniform in $E$. We can then find a function $\omega(n)$ monotonically increasing to $\infty$ and such that $S_{n}^{*}(t) /\left\{R_{n} \omega(n)\right\}^{*}$ still tends to $\infty$ in $E$, and with this function $\omega(n)$ the inequality (6.1.5) is certainly false. This completes the proof of the theorem.

The argument leading to (6.1.3) is obviously crude and there is no reason to expect that it gives the best possible result. It is included here only to show that under very general conditions the maximum $M_{n}(t)$ has no definite order of magnitude for almost every $t$. Under more restrictive conditions, involving third moments, Chung has shown (see his paper in the Transactions of the American Mathematical Soc., 
64 (1948), pp. 205-232) 1 that

$$
\liminf _{n \rightarrow \infty} S_{n}^{*}(t) /\left(\frac{R_{n}}{\log \log R_{n}}\right)^{\frac{1}{2}}=\frac{\pi}{\sqrt{8}}
$$

almost everywhere. (This equality holds, in particular for $\alpha_{1}=\alpha_{2}=\cdots=1$.) Owing to (6.1.4) this leads, under Chung's conditions, to

$$
\lim \inf M_{n}(t) /\left(\frac{R_{n}}{\log \log R_{n}}\right)^{\frac{1}{2}} \leq \frac{\pi}{\sqrt{8}},
$$

an inequality stronger than (6.1.3). Unfortunately, we know nothing about the inequality opposite to $(6.1 .7)^{2}$.

1 We are grateful to Dr. ERDös for calling our attention to Crung's paper. It may be added that (6.1.6) generalizes an earlier result of ErDös who showed that in the case $\alpha_{1}=\alpha_{2}=\cdots=1$ the left side of (6.1.6) is almost everywhere contained between two positive absolute constants.

2 (Added in proof.) Dr. Erdös has communicated us that in the case $\alpha_{1}=\alpha_{2}=\cdots=1$ he can prove that, for every $\varepsilon>0$,

$$
\lim \inf \frac{M_{n}(t)}{n^{\frac{1}{2}-\varepsilon}}>0
$$

almost everywhere, and even a somewhat stronger result. 